\title{
ACC/AATS/AHA/ASE/ASNC/HRS/SCAI/SCCT/SCMR/ STS 2019 appropriate use criteria for multimodality imaging in the assessment of cardiac structure and function in nonvalvular heart disease
}

A report of the American College of Cardiology Appropriate Use Criteria Task Force, American Association for Thoracic Surgery, American Heart Association, American Society of Echocardiography, American Society of Nuclear Cardiology, Heart Rhythm Society, Society for Cardiovascular Angiography and Interventions, Society of Cardiovascular Computed Tomography, Society for Cardiovascular Magnetic Resonance, and the Society of Thoracic Surgeons

Writing Group Members:

\section{Rating Panel Members:}

\section{Appropriate Use Criteria Task} Force:
John U. Doherty, MD, FACC, FAHA, Chair, ${ }^{\text {a }}$ Smadar Kort, MD, FACC, FASE, FAHA, Roxana Mehran, MD, FACC, FSCAI, FAHA, ${ }^{\mathrm{c}}$ Paul Schoenhagen, MD, FAHA, ${ }^{\mathrm{d}}$ and Prem Soman, MD, PhD, FACC

Gregory J. Dehmer, MD, MACC, MSCAI, FACP, FAHA, Moderator, John U. Doherty, MD, FACC, FACP, FAHA, Writing Group Liaison, ${ }^{2}$ Paul Schoenhagen, MD, FAHA, Writing Group Liaison, ${ }^{\mathrm{d}}$ Thomas M. Bashore, MD, FACC, ${ }^{a}$ Nicole M. Bhave, MD, FACC, ${ }^{a}$ Dennis A. Calnon, MD, FACC, FASE, MASNC, FSCCT, ${ }^{\mathrm{e}}$ Blase Carabello, MD, FACC, ${ }^{\mathrm{a}} \mathrm{John}$ Conte, MD, ${ }^{\mathrm{f}}$ Timm Dickfeld, MD, FACC, ${ }^{\mathrm{g}}$ Daniel Edmundowicz, MD, FACC, ${ }^{\text {a }}$ Victor A. Ferrari, MD, FACC, ${ }^{\text {h }}$ Michael E. Hall, MD, FACC, ${ }^{\mathrm{a}}$ Brian Ghoshhajra, MD, MBA, ${ }^{\mathrm{d}}$ Praveen Mehrotra, MD, FACC, ${ }^{\mathrm{a}}$

Tasneem Z. Naqvi, MD, FACC, FASE, ${ }^{\text {a }}$ T. Brett Reece, MD, ${ }^{i}$ Randall C. Starling, MD, FACC, ${ }^{\text {a }}$ Molly Szerlip, MD, MD, FACC, ${ }^{c}$ Wendy S. Tzou, MD, FACC, FHRS ${ }^{j}$ and John B. Wong, MD

John U. Doherty, MD, FACC, FAHA, FACP, Co-Chair, Gregory J. Dehmer, MD, MACC, MSCAI, FAHA, Co-Chair, Steven R. Bailey, MD, FACC, FSCAI, FAHA, Nicole M. Bhave, MD, FACC, Alan S. Brown, MD, FACC, ${ }^{\mathrm{k}}$ Stacie L. Daugherty, MD, FACC, Larry S. Dean, MD, FACC, MSCAI Milind Y. Desai, MBBS, FACC, Claire S. Duvernoy, MD, FACC, Linda D. Gillam, MD, FACC, Robert C. Hendel, MD, FACC, FAHA, ${ }^{\mathrm{k}}$ Christopher M. Kramer, MD, FACC, FAHA, ${ }^{1}$ Bruce D. Lindsay, MD, FACC ${ }^{\mathrm{k}}$ Warren J. Manning, MD, FACC, Manesh R. Patel, MD, FACC, FAHA, ${ }^{\mathrm{m}}$ Ritu Sachdeva, MBBS, FACC, L. Samuel Wann, MD, MACC, ${ }^{k}$ David E. Winchester, MD, FACC, and Michael J. Wolk, MD, MACC ${ }^{\mathrm{k}}$

\footnotetext{
From the american College of Cardiology Representative; ${ }^{\mathrm{b}}$ American Society of Echocardiography Representative; ' Society for Cardiovascular Angiography and Interventions Representative; ${ }^{\mathrm{d}}$ American Society of Nuclear Cardiology Representative; ${ }^{\mathrm{e}}$ Society of Cardiovascular Computed Tomography Representative; ${ }^{\text {f Society }}$ of Thoracic Surgeons Representative; ${ }^{\mathrm{g}} \mathrm{Heart}$ Rhythm Society Representative; ${ }^{\mathrm{h}}$ Society for Cardiovascular Magnetic Resonance Representative; ${ }^{\mathrm{i} A m e r i c a n}$ Association for Thoracic Surgery Representative; ${ }^{\mathrm{j} A m e r i c a n}$ Heart Association Representative; ${ }^{\mathrm{k}}$ Former Task Force member; member during writing effort; ${ }^{\mathrm{l}}$ Former Task Force Cohair; Co-hair during writing effort; ${ }^{\mathrm{m}}$ Former Task Force Chair; Chair during writing effort.This document was approved by the American College of Cardiology Clinical Policy Approval Committee in December 2019.

The American College of Cardiology requests that this document be cited as follows: Doherty JU, Kort S, Mehran R, Schoenhagen P, Soman P. ACC/AATS/AHA/ASE/ ASNC/HRS/SCAI/SCCT/SCMR/STS 2019 appropriate use criteria for multimodality imaging in the assessment of cardiac structure and function in nonvalvular heart disease: a report of the American College of Cardiology Appropriate Use Criteria Task Force, American Association for Thoracic Surgery, American Heart Association, American Society of Echocardiography, American Society of Nuclear Cardiology, Heart Rhythm Society, Society for Cardiovascular Angiography and Interventions, So-
}

ciety of Cardiovascular Computed Tomography, Society for Cardiovascular Magnetic Resonance, and Society of Thoracic Surgeons. J Thorac Cardiovasc Surg. 2019; 157:e153-82

This document has been reprinted in the Journal of the American Society of Echocar diography, Catheterization and Cardiovascular Interventions, the Journal of Cardiovascular Computed Tomography, the Journal of Nuclear Cardiology, and the Journal of Thoracic and Cardiovascular Surgery.

Copies: This document is available on the World Wide Web site of the American College of Cardiology (www.acc.org). For copies of this document, please contact Elsevier Inc Reprint Department via fax (212) 633-3820 or e-mail (reprints@elsevier.com).

Permissions: Multiple copies, modification, alteration, enhancement, and/or distribution of this document are not permitted without the express permission of the American College of Cardiology. Requests may be completed online via the Elsevier site (https://www.elsevier.com/about/our-business/policies/copyright/permissions).

J Thorac Cardiovasc Surg 2019;157:e153-82

$0022-5223 / \$ 36.00$

(C) 2018 by the American College of Cardiology Foundation

https://doi.org/10.1016/j.jtcvs.2018.12.061 
See Commentary page 1465 .

\section{TABLE OF CONTENTS}

Abstract. . . . . . . . . . . . . . . . . . e154

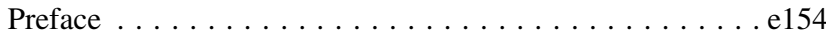

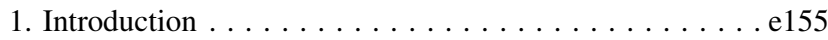

Table A. Stages of Valvular Heart Disease . . . . . . . e155

Table B. Stages of Heart Failure . . . . . . . . . . . . . . . . . . . . . . . . . . . . . . . . . . . .

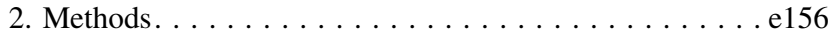

Indication Development . . . . . . . . . . . . . . . . . . . . . . . . 156

3. General Assumptions. . . . . . . . . . . . . . . . e157

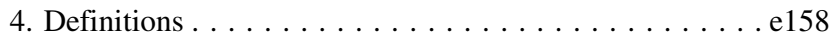

5. Abbreviations . . . . . . . . . . . . . . . e159

6. Multimodality Imaging in Nonvalvular Heart Disease:

Appropriate Use Criteria (by Indication) . . . . . . . . . . . e160

6.1. Initial Evaluation of Cardiac Structure and Function . e160

Table 1. Initial Evaluation of an Asymptomatic Patiente160

Table 2. Initial Evaluation of a Patient With Clinical

Signs and/or Symptoms of Heart Disease . e161

6.2. Evaluation of Cardiac Structure and Function in

Patients Who Had Prior Testing . . . . . . . . . . . . e164

Table 3. Sequential or Follow-up Testing to Clarify

Initial Diagnostic Testing. . . . . . . . . . . e164

Table 4. Sequential or Follow-up Testing:

Asymptomatic or Stable Symptoms. . . . . . e165

Table 5. Sequential or Follow-up Testing: New or

Worsening Symptoms or to Guide Therapy e166

6.3. Evaluation of Cardiac Structure and Function in

Patients Undergoing Transcatheter Intervention for

Structural Heart Disease . . . . . . . . . . . . . . e167

Table 6. Imaging for the Evaluation of TIA or Ischemic

Stroke ................ . . 167

6.3.1. Imaging for the evaluation of patent foramen ovale or atrial septal defect . . . . . . . e168

Table 7. Preprocedural Evaluation for Closure of PFO

or Atrial Septal Defect . . . . . . . . e168

Table 8. Intraprocedural Guidance for Closure of PFO

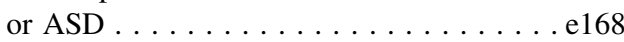

Table 9. Assessment Following Closure of PFO or

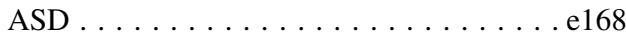

6.3.2. Imaging for the evaluation of left atrial appendage occlusion device. . . . . . . e e169

Table 10. Preprocedural Evaluation for LAA

Occlusion . . . . . . . . . . . e169

Table 11. Intraprocedural Guidance for LAA

Occlusion . . . . . . . . . . . . e 169

Table 12. Assessment Following LAA Occlusion. e169

7. Discussion . . . . . . . . . . . . . . . . e170

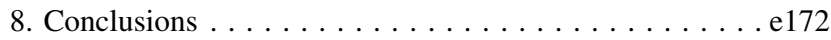

ACC President and Staff . . . . . . . . . . . . . . e172

References... . . . . . . . . . . . . . . . . . e172

Appendix A. Relationships With Industry and

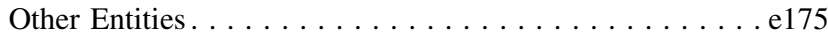

\section{ABSTRACT}

This document is the second of 2 companion appropriate use criteria (AUC) documents developed by the
American College of Cardiology, American Association for Thoracic Surgery, American Heart Association, American Society of Echocardiography, American Society of Nuclear Cardiology, Heart Rhythm Society, Society for Cardiovascular Angiography and Interventions, Society of Cardiovascular Computed Tomography, Society for Cardiovascular Magnetic Resonance, and Society of Thoracic Surgeons. The first document ${ }^{1}$ addresses the evaluation and use of multimodality imaging in the diagnosis and management of valvular heart disease, whereas this document addresses this topic with regard to structural (nonvalvular) heart disease. While dealing with different subjects, the 2 documents do share a common structure and feature some clinical overlap. The goal of the companion AUC documents is to provide a comprehensive resource for multimodality imaging in the context of structural and valvular heart disease, encompassing multiple imaging modalities.

Using standardized methodology, the clinical scenarios (indications) were developed by a diverse writing group to represent patient presentations encountered in everyday practice and included common applications and anticipated uses. Where appropriate, the scenarios were developed on the basis of the most current American College of Cardiology/American Heart Association Clinical Practice Guidelines.

A separate, independent rating panel scored the 102 clinical scenarios in this document on a scale of 1 to 9 . Scores of 7 to 9 indicate that a modality is considered appropriate for the clinical scenario presented. Midrange scores of 4 to 6 indicate that a modality may be appropriate for the clinical scenario, and scores of 1 to 3 indicate that a modality is considered rarely appropriate for the clinical scenario.

The primary objective of the AUC is to provide a framework for the assessment of these scenarios by practices that will improve and standardize physician decision making. AUC publications reflect an ongoing effort by the American College of Cardiology to critically and systematically create, review, and categorize clinical situations in which diagnostic tests and procedures are utilized by physicians caring for patients with cardiovascular diseases. The process is based on the current understanding of the technical capabilities of the imaging modalities examined.

\section{PREFACE}

Structural and valvular heart disease (VHD) encompass a significant proportion of cardiovascular disease conditions. Initial diagnosis and subsequent follow-up frequently rely on imaging with more than 1 imaging modality. Rapidly evolving less-invasive and transcatheter treatment options have fueled the need for precise preprocedural and intraprocedural anatomic and functional imaging. 
The publication of appropriate use criteria (AUC) reflects 1 of several ongoing efforts by the American College of Cardiology (ACC) and its partners to assist clinicians who are caring for patients with cardiovascular diseases and support high-quality cardiovascular care. The ACC/American Heart Association Clinical Practice Guidelines provide a foundation for summarizing evidence-based cardiovascular care and, when evidence is lacking, expert consensus opinion that is approved in review by the ACC and American Heart Association. However, in many areas, variability remains in the use of cardiovascular imaging modalities, raising questions of overuse or underuse. The AUC provide a practical standard upon which to assess and better understand variability.

We are grateful to the writing committee for the development of the overall structure of the document and clinical scenarios and to the rating panel-a professional group with a wide range of skills and insights - for their thoughtful deliberation of the merits of multimodality imaging for various clinical scenarios. A special thanks to Dr. Gregory J. Dehmer for serving as an expert moderator at our inperson rating panel meeting. We would also like to thank the AUC Task Force members, who provided insight and guidance, and the ACC staff-especially María Velásquez-for their skilled support in the generation of this document.

\section{John U. Doherty, MD, FACC, FAHA, FACP Chair, Multimodality Imaging in Nonvalvular Heart Disease Writing Group Co-Chair, Appropriate Use Criteria Task Force}

\section{INTRODUCTION}

Improvements in noninvasive cardiovascular imaging technologies and their broader application to cardiovascular diagnosis and therapy have led to a dizzying array of imaging options for the clinician. The strengths and limitations of various modalities are increasingly a body of knowledge that may be unfamiliar to general clinicians, who are, at the same time, striving to be responsible stewards of medical resources. The Appropriate Use methodology has evolved from the evaluation of single modalities of imaging to a diagnosis-based and patient-centered approach evaluating multiple diagnostic options in the assessment and care of our patients.

Through efforts to derive maximal value from imaging, the rate of imaging volume growth in Medicare has been slowing. Still, the armamentarium of noninvasive diagnostic tools has expanded greatly, offering a variety of new and more sophisticated imaging techniques. As imaging technology and clinical applications continue to advance, the healthcare community must understand how best to incorporate these technologies into daily clinical care and how to choose between new and established imaging technologies.

Proper diagnosis of structural heart disease has become critical as numerous catheter-based interventions are now available as less-invasive therapeutic options. For the purpose of this document, structural disease is used more broadly and includes heart failure and diseases of the aorta and pericardium, in essence, any disorder in which there is an abnormality of cardiac structure or function, excluding valvular diseases.

Using standardized methodology, the clinical scenarios (indications) in this document were developed by a diverse writing group to represent patient presentations encountered in everyday practice and were evaluated and rated by a separate, independent rating panel.

Because there is significant clinical overlap between structural and valvular heart disease, separating the indications in the 2 AUC documents is somewhat arbitrary. The writing group therefore deliberately followed a common structure in creating the companion documents on structural heart disease and VHD.

Specifically, this document is organized into 4 sections and 8 tables. Section 4 provides definitions of key concepts in structural heart disease, with Table A defining the stages of valvular heart disease and Table B defining the stages of heart failure. Section 6.1. describes scenarios of initial evaluation with no prior imaging. Table 1 lists scenarios for the asymptomatic patient, whereas Table 2 lists scenarios for the symptomatic patient. Section 6.2. describes scenarios in which prior imaging has been performed and sequential evaluation required. Table 3 rates scenarios in which additional testing is used to clarify the initial diagnosis. This is meant to span the period of initial evaluation, with further

TABLE A. Stages of valvular heart disease

\begin{tabular}{|c|c|c|}
\hline Stage & Definition & Description \\
\hline A & At risk & $\begin{array}{l}\text { Patients with risk factors for development } \\
\text { of VHD }\end{array}$ \\
\hline B & Progressive & $\begin{array}{l}\text { Patients with progressive VHD (mild to } \\
\text { moderate severity and asymptomatic) }\end{array}$ \\
\hline $\mathrm{C}$ & $\begin{array}{l}\text { Asymptomatic } \\
\text { severe }\end{array}$ & $\begin{array}{l}\text { Asymptomatic patients who meet criteria } \\
\text { for severe VHD: } \\
\text { C1: Asymptomatic patients with } \\
\text { severe VHD in whom the left or } \\
\text { right ventricle remains } \\
\text { compensated } \\
\text { C2: Asymptomatic patients with } \\
\text { severe VHD with decompensation } \\
\text { of the left or right ventricle }\end{array}$ \\
\hline D & $\begin{array}{l}\text { Symptomatic } \\
\text { severe }\end{array}$ & $\begin{array}{l}\text { Patients who have developed symptoms } \\
\text { as a result of severe VHD }\end{array}$ \\
\hline
\end{tabular}

$V H D$, Valvular heart disease.Reproduced from Nishimura et al. ${ }^{8}$ 
TABLE B. Stages of Heart Failure

\begin{tabular}{ll}
\hline Stage & \multicolumn{1}{c}{ Definition } \\
\hline Stage A & $\begin{array}{c}\text { Patients with risk factors for heart failure but } \\
\text { without structural disease or symptoms (eg, } \\
\text { patient with hypertension but without left } \\
\text { ventricular hypertrophy). }\end{array}$ \\
Stage B & $\begin{array}{l}\text { Patient with structural disease but no symptoms } \\
\text { (eg, asymptomatic left ventricular } \\
\text { hypertrophy) }\end{array}$ \\
Stage C & Current or prior symptoms of heart failure \\
Stage D & Drug-refractory heart failure \\
\hline
\end{tabular}

testing performed as needed to establish the diagnosis and guide therapy. This may be more detailed evaluation after the identification of structural heart disease by initial imaging that has not proved definitive either in diagnosis or prognosis or in directing therapy. Table 4 describes scenarios in which additional testing is used in the context of clinical follow-up after initial diagnosis in the asymptomatic patient. This may be done to assess the response to therapy or the stability of the asymptomatic patient in whom structural heart disease has been identified by initial imaging. In this case the imaging modality may be the same as that of the initial study and is used to assess stability and/or guide therapy. Table 5 describes scenarios in which follow-up testing is done in the symptomatic patient. It encompasses follow-up imaging after the identification of structural heart disease in the face of new or worsening symptoms. Section 6.3. evaluates transcatheter intervention for structural heart disease (Tables 6-12). Table 6 and Tables 7-9 evaluate diagnosis and imaging support for transient ischemic cerebral attacks and identify patent foramen ovale preprocedural, intraprocedural, and postprocedural scenarios. Tables 10-12 are further divided into preprocedural, intraprocedural, and postprocedural indications. For these indications, imaging support for left atrial appendage occlusion assumes the intervention's clinical appropriateness.

\section{METHODS}

\section{Indication Development}

This document covers a wide array of methods for treating structural heart disease. A standardized approach was used to create different categories of indications, with the goal of capturing actual clinical scenarios, yet without making the list of indications excessively long. Indications were created to represent most of the possible treatment approaches for structural heart disease, rather than limiting the AUC to indications for which evidence was available.

To identify and categorize the indications, a writing group was formed that comprised structural heart disease experts representing a variety of organizations and societies. Wherever possible during the writing process, the group members would map the indications to relevant clinical practice guidelines and key publications or references (see Online Appendix). Once the indications were formed, they were reviewed and critiqued by the parent AUC Task Force and by numerous external reviewers, including interventionalists, surgeons, radiologists, imagers, and generalists. After the writing group incorporated this initial feedback, the indications were sent to an independent rating panel comprising additional experts in the structural heart disease realm. The indications were then sent back to the writing group for additional vetting. Imaging for each indication was then rated and classified as Appropriate (A), May Be Appropriate (M), or Rarely Appropriate (R) on the basis of these multiple rounds of review and revision.

A detailed description for the rating of imaging modalities is found in a previous publication, ACCF Proposed Method for Evaluating the Appropriateness of Cardiovascular Imaging, ${ }^{2}$ as well as the updated version, ACC Appropriate Use Criteria Methodology: 2018 Update. ${ }^{3}$ Briefly, this process combines evidence-based medicine and practice experience, and engages a rating panel in a modified Delphi exercise. Other steps include convening a formal writing group with diverse expertise in structural heart disease, circulating the indications for external review prior to sending them to the rating panel, ensuring an appropriate balance of expertise and practice area in the rating panel, developing a standardized rating package that includes relevant evidence, and establishment of formal roles for facilitating panel interaction at the face-to-face meeting.

The rating panel first evaluated the indications independently. Then the panel was convened for a face-to-face meeting to discuss each indication. At this meeting, panel members were given their scores and a blinded summary of their peers' scores. After the meeting, panel members were asked to provide their final scores for each indication independently.

Although panel members were not provided explicit cost information to help determine their Appropriate Use ratings, they were asked to implicitly consider cost as an additional factor in their evaluation of Appropriate Use. In rating these criteria, the AUC Rating Panel was asked to assess whether the use of the test for each indication is Appropriate, May Be Appropriate, or Rarely Appropriate, and was provided the following definition of Appropriate Use:

An appropriate imaging study is one in which the expected incremental information, combined with clinical judgment, exceeds the expected negative consequences* by a sufficiently wide margin for a specific indication that the procedure is generally considered acceptable care and a reasonable approach for the indication.

The rating panel scored each indication as follows:

Median Score 7 to 9: Appropriate test for specific indication (test is generally acceptable and is a reasonable approach for the indication).

An appropriate option for management of patients in this population due to benefits generally outweighing risks; an effective option for individual care plans, although not always necessary depending on physician judgment and patient-specific preferences (i.e., procedure is generally acceptable and is generally reasonable for the indication).

Median Score 4 to 6: May Be Appropriate test for specific indication (test may be generally acceptable and may be a reasonable approach for the indication). May Be Appropriate also implies that more research and/ or patient information is needed to classify the indication definitively.

At times an appropriate option for management of patients in this population due to variable evidence or agreement regarding the benefit-risk ratio, potential benefit based on practice experience in the absence of evidence, and/or variability in the population; effectiveness for individual care must be determined by a patient's

\footnotetext{
* Negative consequences include the risks of the procedure (eg, radiation or contrast exposure) and the downstream impact of poor test performance such as delay in diagnosis (false negatives) or inappropriate diagnosis (false positives).
} 
physician in consultation with the patient on the basis of additional clinical variables and judgment along with patient preferences (i.e., procedure may be acceptable and may be reasonable for the indication).

Median Score 1 to 3: Rarely Appropriate test for specific indication (test is not generally acceptable and is not a reasonable approach for the indication).

Rarely an appropriate option for management of patients in this population due to the lack of a clear benefit/risk advantage; rarely an effective option for individual care plans; exceptions should have documentation of the clinical reasons for proceeding with this care option (i.e., procedure is not generally acceptable and is not generally reasonable for the indication).

The division of the numerical scores into 3 levels of appropriateness is somewhat arbitrary, and the numeric designations should be viewed as a continuum. Further, clinical opinions may vary for particular clinical scenarios, such that scores in the intermediate level of appropriate use were labeled "May Be Appropriate," as critical patient or research data may be lacking or discordant. This designation should be a prompt to the field to carry out definitive research investigation whenever possible. It is anticipated that the AUC reports will continue to be revised as further data are generated and information from implementation of the criteria is accumulated.

To prevent bias in the scoring process, the rating panel was deliberately assembled to include a minority of specialists in structural heart disease. While offering important clinical and technical insights, specialists might have a natural tendency to rate the indications within their specialty as more appropriate than would nonspecialists. In addition, care was taken in providing objective, unbiased information, including clinical practice guidelines and key references, to the rating panel.

The level of agreement among panelists as defined by RAND ${ }^{4}$ was analyzed on the basis of the BIOMED rule for a panel of 14 to 16 members. As such, agreement was defined as an indication where 4 or fewer panelists' ratings fell outside the 3-point region containing the median score.

Disagreement was defined as when at least 5 panelists' ratings fell in both the Appropriate and the Rarely Appropriate categories. Any indication having disagreement was categorized as May Be Appropriate regardless of the final median score.

\section{GENERAL ASSUMPTIONS}

1. This document will address the use of multimodality imaging for evaluation of cardiac structure and function focusing on nonvalvular structural disease and interventions. The companion document evaluates valvular diseases as well as percutaneous interventions used for their treatment.

2. Indication ratings contained herein supersede the ratings of similar indications contained in previous AUC documents.

3. Evaluation of all indications pertains only to nonurgent clinical circumstances.

4. A qualified clinician has obtained a complete clinical history and performed a physical examination so that the clinical status of the patient can be assumed to be valid as stated in the indication. Example: An asymptomatic patient is truly asymptomatic and sufficient questioning has been undertaken for the condition in question.

5. All patients are receiving optimal standard care, including clinical practice guideline-based risk factor modification, primary and secondary prevention of ischemic heart disease, or treatment of heart failure, unless it is specifically noted.

6. The indications are, at times, purposely broad to cover an array of cardiovascular signs and symptoms and to account for the ordering physician's best judgment regarding the presence of cardiovascular abnormalities. Additionally, there are likely clinical scenarios that are not covered in this document.

7. If the reason for a test can be assigned to more than 1 indication, the reason is classified under the most clinically significant indication.

8. Testing modalities are rated for their level of appropriateness specific to clinical scenarios rather than a forced rank-order comparison against other testing modalities. The goal of this document is to identify any and all tests that are considered reasonable for a given clinical indication. The goal of this document is the determination of the range of modalities that may or may not be reasonable for specific indications rather than determination of a single best test for each indication or a rank order. As such, more than 1 test type may be considered Appropriate, May Be Appropriate, or Rarely Appropriate for any given clinical indication.

9. If more than one modality falls into the same Appropriate Use category, physician judgment and available local expertise should be used to determine choice of test.

10. The appropriate use of testing has the potential to impact clinical decision making and to direct therapeutic interventions.

11. Patients are suitable candidates for the procedure after consideration of procedural risk. Unless explicitly stated, it is presumed that patients presenting for a specific clinical indication are potential candidates for all of the tests to be rated and do not present with strong contraindications that preclude them from being tested (eg, renal dysfunction, presence of an implanted device).

12. Risk benefit: Overall patients' representation as described by age and other clinical factors was used in the risk/benefit estimate. Each modality considered in this document has inherent risks that may include but are not limited to radiation exposure, contrast sensitivity, other bodily injury, and interpretation 
errors. For any test, there may be certain patient populations who are more susceptible to its known risks who are not specifically captured in the indications but deserve consideration when rating. Such risks should be viewed "on balance" and not used as justification to systematically reduce the level of appropriateness of a particular test relative to other tests. (eg, tests that expose the patient to ionizing radiation should not necessarily receive a lower score than those that do not). Thus, a given modality should be weighed specifically in the context of the clinical scenario, with the potential harm considered relative to the potential benefit gained.

13. Radiation safety: No clinical evidence to date unequivocally supports the notion that low-dose ionizing radiation at the levels used in medical imaging is associated with increased long-term risk of malignancy. In a conservative approach, many experts in the field have adopted the linear no-threshold hypothesis, which assumes a linear relationship between radiation dose and risk of malignancy irrespective of radiation dose magnitude. Accordingly, the following radiation safety principles should be applied to all testing involving ionizing radiation:

a. Clinical benefit should be As High As Reasonably Achievable, embracing the guiding principle that testing should be performed on cohorts that are most likely to experience a net benefit.

b. Radiation exposure should be As Low As Reasonably Achievable (ALARA). ALARA should be used to guide test choice and the imaging protocol. Implicit in the ALARA principle is that the use of tests involving ionizing radiation should be minimized in vulnerable populations such as younger patients, and that optimal test procedures are utilized to perform the test at the lowest possible radiation dose while preserving image quality and information output.

c. Consider a patient's exposure to ionizing radiation for noncardiac diseases. These principles and their implications for care have recently been evaluated in the $2018 \mathrm{ACC} / \mathrm{HRS} / \mathrm{NASCI} / \mathrm{SCAI} / \mathrm{SCCT}$ Expert Consensus Document on Optimal Use of Ionizing Radiation in Cardiovascular Imaging. ${ }^{5}$

14. Selection of patients for and monitoring of patients during and after contrast administration are assumed to be in accord with published standards when available.

15. Cost: Clinical benefit should always be considered first, and cost should be considered in relationship to these benefits when determining net value. For example, a procedure with moderate clinical effi- cacy for a given AUC indication should not be scored as more appropriate than a procedure with a high clinical efficacy solely because of lower cost. Value may be informed by multiple measures of potential economic impact such as: a) induced downstream or layered testing rates; b) comparative cost savings or minimization for diagnostic or nearterm follow-up, c) cost to reduce adverse outcomes (eg, cost for hospitalization averted); d) cost for life years gained.

16. All tests and procedures are presumed to be performed and interpreted by qualified individuals in a facility that is in compliance with national standards for performing such imaging studies or procedures. Therefore, the level of appropriateness does not consider issues of local availability or skill in the rating of any modality.

17. Time biases in available data: Newer technologies should not be considered necessarily more or less appropriate than older technologies. Apparent differences in diagnostic accuracy and risk stratification between older and newer techniques may not be accurate, especially when the techniques are not compared directly or when historical data are utilized. As treatment paradigms evolve, diagnosis may occur at earlier stages of disease, posing unique challenges for comparison of the performance of diagnostic modalities used at different stages of the disease process owing to time lag bias.

18. Patients are suitable candidates for the procedure, with suitable procedural risk.

\section{DEFINITIONS}

\section{Family History}

In this document, the term "family history" refers to first-degree relatives only.

\section{Symptomatic}

A patient is deemed to be symptomatic when he/ she exhibits typical signs and/or symptoms (eg, for congestive heart failure, symptoms such as dyspnea, rales, edema, and limited exercise capacity).

\section{Asymptomatic}

Patient is deemed asymptomatic when he/she exhibits none of the typical symptoms.

\section{Low, Moderate, and High Pretest Probability}

As defined by the "2013 ACC/AHA/AATS/PCNA/ SCAI/STS Focused Update of the Guideline for the Diagnosis and Management of Patients with 
Stable Ischemic Heart Disease", 6 Low pretest probability indicates $<10 \%$ probability of disease prior to the test under consideration. Moderate pretest probability is a range of $10 \%$ to $90 \%$ pretest probability. High pretest probability is a $>90 \%$ likelihood of the presence of the disease entity under question prior to any testing.

\section{Clinically Significant}

An abnormality that, if left untreated, can or will lead to functional impairment or death.

6. Mild, Moderate, and Severe Valvular Disease

As defined by the "2017 AHA/ACC Focused Update of the 2014 AHA/ACC Guideline for the Management of Patients with Valvular Heart Disease", 7,8

\section{Stages of VHD}

VHD as defined by the "2017 AHA/ACC Focused Update of the 2014 AHA/ACC Guideline for the Management of Patients with Valvular Heart Disease $^{, 7,8}$ (Table A).

\section{Uninterpretable or Technically Limited Images}

Images that are not of diagnostic quality despite performance of the study by a skilled sonographer, technician, or other provider using appropriate equipment. This may be due to patient-related factors such as body habitus or motion artifact.

\section{Concomitant Coronary Artery Disease}

Term used when coronary artery disease does not explain most of the clinical symptomology of the patient but does occur in conjunction with another disease entity.

\section{Frequent Ventricular Premature Contractions}

Ventricular premature contractions occurring more frequently than 30 times per hour or occurring in a pattern of bigeminy, trigeminy, or runs of ventricular tachycardia.

\section{Infrequent Atrial Premature Complexes}

Atrial premature complexes occurring less than 30 times per hour or less than once per minute.

\section{Nonsustained Ventricular Tachycardia}

Ventricular arrhythmia of 3 or more consecutive complexes but lasting $<30$ seconds in duration at a rate $>100$ bpm.

\section{Sustained Ventricular Tachycardia}

Ventricular tachycardia lasting more than 30 seconds or requiring therapy because of hemodynamic compromise in $<30$ seconds.

\section{Syncope}

Transient loss of consciousness due to global cerebral hypoperfusion characterized by rapid onset, short duration, and spontaneous complete recovery; not lightheadedness or dizziness alone.

\section{Presyncope}

Near loss of consciousness.

\section{Heart Failure}

Signs and symptoms explainable on the basis of systolic or diastolic dysfunction.

\section{Heart Failure Stages A, B, C, and D}

Heart failure as defined by the " 2009 Focused Update Incorporated into the ACC/AHA 2005 Guidelines for the Diagnosis and Management of Heart Failure in Adults", (Table B).

\section{Indication}

Synonymous with "scenario." A set of patientspecific conditions defines "indication." The term "clinical indication" does not necessarily imply that testing is warranted. In other words, for some clinical indications, all modalities may be rated as Rarely Appropriate.

\section{ABBREVIATIONS}

3D $=3$-dimensional

ANG = invasive coronary angiography/ventriculography/ aortography

$\mathrm{AUC}=$ appropriate use criteria

Cardiac $\mathrm{MR}=$ cardiovascular magnetic resonance imaging

$\mathrm{CRT}=$ cardiac resynchronization therapy

$\mathrm{CT}=$ computed tomography

DSE $=$ dobutamine stress echocardiography

FDG-PET = fluorodeoxyglucose-positron emission tomography

MPI = myocardial perfusion imaging

$\mathrm{RVG}=$ radionuclide ventriculography

SPECT $=$ single-photon emission computed tomography

TEE $=$ transesophageal echocardiography

$\mathrm{TTE}=$ transthoracic echocardiography 


\section{MULTIMODALITY IMAGING IN NONVALVULAR HEART DISEASE: APPROPRIATE USE CRITERIA (BY INDICATION) \\ 6.1. Initial Evaluation of Cardiac Structure and Function}

TABLE 1. Initial evaluation of an asymptomatic patient

\begin{tabular}{|c|c|c|c|c|c|c|c|c|}
\hline Indication & $\begin{array}{l}\text { TTE (With or } \\
\text { Without 3D; } \\
\text { With Contrast } \\
\text { as Needed) }\end{array}$ & $\begin{array}{c}\text { TEE } \\
\text { (With or } \\
\text { Without 3D) }\end{array}$ & $\begin{array}{l}\text { Stress } \\
\text { Echo* }\end{array}$ & $\begin{array}{c}\text { Strain/Strain } \\
\text { Rate Imaging by } \\
\text { Speckle or Tissue } \\
\text { Doppler }\end{array}$ & RVG & $\begin{array}{l}\text { MPI } \\
\text { (SPECT/ } \\
\text { PET) }\end{array}$ & CMR & CT \\
\hline $\begin{array}{l}\text { 1. Initial cardiac evaluation of a known systemic, } \\
\text { congenital, or acquired disease that could be } \\
\text { associated with structural heart disease }\end{array}$ & $9(\mathrm{~A})$ & $2(R)$ & $2(R)$ & $5(\mathrm{M})$ & $1(R)$ & $2(R)$ & $5(\mathrm{M})$ & $2(R)$ \\
\hline $\begin{array}{l}\text { 2. Screening evaluation for structure and function in } \\
\text { first-degree relatives of a patient with an } \\
\text { inherited cardiomyopathy }\end{array}$ & 9 (A) & $1(R)$ & 2 (R) & $4(M)$ & $1(R)$ & $1(R)$ & $5(M)$ & $2(R)$ \\
\hline $\begin{array}{l}\text { 3. Initial evaluation prior to exposure to } \\
\text { medications/radiation that could result in } \\
\text { cardiotoxicity/heart failure }\end{array}$ & $9(\mathrm{~A})$ & $2(R)$ & $2(R)$ & 7 (A) & $7(A)$ & $2(R)$ & $5(M)$ & 2 (R) \\
\hline $\begin{array}{l}\text { 4. Evaluation of the ascending aorta in the setting } \\
\text { of a known or suspected connective tissue } \\
\text { disease or genetic condition that predisposes to } \\
\text { aortic aneurysm or dissection (e.g., Marfan } \\
\text { syndrome) }\end{array}$ & $8(A)$ & $5(M)$ & $1(\mathrm{R})$ & $1(R)$ & $1(R)$ & $1(\mathrm{R})$ & $8(A)$ & $8(A)$ \\
\hline $\begin{array}{l}\text { 5. Screening evaluation in relatives of a patient with } \\
\text { known aortic aneurysm or dissection }\end{array}$ & $8(A)$ & $3(\mathrm{R})$ & $1(R)$ & $1(R)$ & $1(R)$ & $1(R)$ & 7 (A) & 7 (A) \\
\hline $\begin{array}{l}\text { 6. Preparticipation athlete assessment in a patient } \\
\text { with no symptoms, normal examination, and no } \\
\text { family history of inheritable heart disease }\end{array}$ & $3(\mathrm{R})$ & $1(\mathrm{R})$ & $1(R)$ & $1(R)$ & $1(R)$ & 1 (R) & $1(\mathrm{R})$ & 1 (R) \\
\hline $\begin{array}{l}\text { 7. Preparticipation assessment of an asymptomatic } \\
\text { athlete with } \geq 1 \text { of the following: abnormal } \\
\text { examination, abnormal ECG, or definite (or high } \\
\text { suspicion for) family history of inheritable heart } \\
\text { disease }\end{array}$ & $9(\mathrm{~A})$ & $2(R)$ & $4(\mathrm{M})$ & $4(M)$ & $1(\mathrm{R})$ & $2(R)$ & $5(M)$ & $4(M)$ \\
\hline $\begin{array}{l}\text { 8. Evaluation of suspected pulmonary arterial } \\
\text { hypertension, including evaluation of right } \\
\text { ventricular function and estimated pulmonary } \\
\text { artery pressure in a patient at risk for developing } \\
\text { pulmonary arterial hypertension }\end{array}$ & $9(\mathrm{~A})$ & $2(R)$ & $3(R)$ & $2(R)$ & $1(R)$ & $1(R)$ & $4(\mathrm{M})$ & 4 (M) \\
\hline
\end{tabular}

TTE, Transthoracic echocardiography; $3 D$, 3-dimensional; TEE, transesophageal echocardiography; Echo, echocardiography; $R V G$, radionuclide ventriculography; $M P I$, myocardial perfusion imaging; SPECT, single-photon emission computed tomography; $P E T$, positron emission tomography; $C M R$, cardiovascular magnetic resonance imaging; $C T$, computed tomography; $A$, appropriate; $R$, rarely appropriate; $M$, may be appropriate; $E C G$, electrocardiogram. *Stress echo comprises exercise stress echocardiography and dobutamine stress echocardiography. 
TABLE 2. Initial evaluation of a patient with clinical signs and/or symptoms of heart disease

\begin{tabular}{|c|c|c|c|c|c|c|c|c|c|c|c|}
\hline Indication & $\begin{array}{l}\text { TTE (With or } \\
\text { Without 3D; } \\
\text { With Contrast as } \\
\text { Needed) }\end{array}$ & $\begin{array}{c}\text { TEE } \\
\text { (With or } \\
\text { Without 3D) }\end{array}$ & $\begin{array}{l}\text { Stress } \\
\text { Echo* }\end{array}$ & $\begin{array}{l}\text { Strain/Strain } \\
\text { Rate Imaging } \\
\text { by Speckle or } \\
\text { Tissue Doppler }\end{array}$ & $\begin{array}{c}\text { F-18 FDG } \\
\text { PET }\end{array}$ & $\begin{array}{l}\text { TC- } \\
99 \mathrm{~m} \\
\text { PYP }\end{array}$ & $\begin{array}{l}\text { MPI } \\
\text { (SPECT/ } \\
\text { PET) }\end{array}$ & CMR & $\mathbf{C T} \dagger$ & ANG & RVG \\
\hline $\begin{array}{l}\text { 9. Initial evaluation when } \\
\text { symptoms or signs } \\
\text { suggest heart disease }\end{array}$ & $9(A)$ & $3(\mathrm{R})$ & $5(\mathrm{M})$ & $5(M)$ & $2(R)$ & $2(R)$ & $5(M)$ & $5(\mathrm{M})$ & $4(M)$ & $3(R)$ & $4(\mathrm{M})$ \\
\hline \multicolumn{12}{|c|}{ Arrhythmias or Conduction Disorders } \\
\hline 10. Newly diagnosed LBBB & $7(\mathrm{~A})$ & $2(R)$ & $4(M)$ & $4(\mathrm{M})$ & $1(\mathrm{R})$ & $1(R)$ & $5(M)$ & $4(\mathrm{M})$ & $3(R)$ & $2(\mathrm{R})$ & $3(\mathrm{R})$ \\
\hline 11. Newly diagnosed RBBB & $5(\mathrm{M})$ & $1(\mathrm{R})$ & $3(\mathrm{R})$ & $2(R)$ & $1(\mathrm{R})$ & $1(\mathrm{R})$ & $2(R)$ & $2(R)$ & $2(R)$ & $1(R)$ & $2(R)$ \\
\hline $\begin{array}{l}\text { 12. Frequent VPCs without } \\
\text { other evidence of heart } \\
\text { disease }\end{array}$ & $7(A)$ & $2(\mathrm{R})$ & $3(\mathrm{R})$ & $2(\mathrm{R})$ & $1(\mathrm{R})$ & $1(\mathrm{R})$ & $3(\mathrm{R})$ & $4(\mathrm{M})$ & $3(R)$ & $2(\mathrm{R})$ & $2(\mathrm{R})$ \\
\hline 13. Nonsustained VT & $8(A)$ & $2(\mathrm{R})$ & $5(\mathrm{M})$ & $4(\mathrm{M})$ & $2(\mathrm{R})$ & $1(\mathrm{R})$ & $5(\mathrm{M})$ & $5(\mathrm{M})$ & $3(\mathrm{R})$ & $3(\mathrm{R})$ & $3(\mathrm{R})$ \\
\hline 14. Sustained VT or VF & $9(\mathrm{~A})$ & $2(R)$ & $6(\mathrm{M})$ & $3(\mathrm{R})$ & 2 (R) & 2 (R) & $6(M)$ & $6(\mathrm{M})$ & $6(M)$ & 7 (A) & $4(M)$ \\
\hline $\begin{array}{l}\text { 15. Evaluation of the patient } \\
\text { with episodes of SVT } \\
\text { without other evidence of } \\
\text { heart disease }\end{array}$ & $6(\mathrm{M})$ & $2(R)$ & 2 (R) & $1(\mathrm{R})$ & 1 (R) & 1 (R) & $1(R)$ & 2 (R) & $1(R)$ & $1(R)$ & 1 (R) \\
\hline $\begin{array}{l}\text { 16. Atrial fibrillation/flutter } \\
\text { (not for purposes of } \\
\text { precardioversion } \\
\text { evaluation) }\end{array}$ & $8(A)$ & $2(R)$ & $6(\mathrm{M})$ & $3(\mathrm{R})$ & $1(\mathrm{R})$ & $1(\mathrm{R})$ & $4(\mathrm{M})$ & 3 (R) & $3(R)$ & $2(R)$ & $2(R)$ \\
\hline \multicolumn{12}{|c|}{ Palpitations/Presyncope/Syncope } \\
\hline $\begin{array}{l}\text { 17. Clinical symptoms or signs } \\
\text { consistent with a cardiac } \\
\text { diagnosis known to cause } \\
\text { presyncope/syncope } \\
\text { (including but not limited } \\
\text { to hypertrophic } \\
\text { cardiomyopathy and HF) }\end{array}$ & 9 (A) & $3(\mathrm{R})$ & $5(\mathrm{M})$ & $4(\mathrm{M})$ & $2(R)$ & $1(\mathrm{R})$ & $5(\mathrm{M})$ & 7 (A) & $5(\mathrm{M})$ & $3(\mathrm{R})$ & 3 (R) \\
\hline $\begin{array}{l}\text { 18. Palpitations without other } \\
\text { symptoms or signs of } \\
\text { cardiovascular disease }\end{array}$ & $6(\mathrm{M})$ & $1(R)$ & $2(R)$ & $2(R)$ & $1(R)$ & $1(R)$ & $2(R)$ & $2(R)$ & $1(R)$ & 1 (R) & 1 (R) \\
\hline $\begin{array}{l}\text { 19. Presyncope without other } \\
\text { symptoms or signs of } \\
\text { cardiovascular disease }\end{array}$ & $7(A)$ & $1(R)$ & $1(R)$ & 2 (R) & 1 (R) & $1(R)$ & 1 (R) & 2 (R) & $1(R)$ & 1 (R) & $1(\mathrm{R})$ \\
\hline $\begin{array}{l}\text { 20. Syncope without other } \\
\text { symptoms or signs of } \\
\text { cardiovascular disease }\end{array}$ & $8(A)$ & $2(R)$ & $4(\mathrm{M})$ & $2(R)$ & $1(\mathrm{R})$ & $1(\mathrm{R})$ & $4(\mathrm{M})$ & $3(\mathrm{R})$ & $2(R)$ & 2 (R) & $2(R)$ \\
\hline \multicolumn{12}{|c|}{ Hypotension or Hemodynamic Instability } \\
\hline $\begin{array}{l}\text { 21. Hypotension or } \\
\text { hemodynamic instability } \\
\text { of uncertain or suspected } \\
\text { cardiac etiology }\end{array}$ & $8(A)$ & $4(M)$ & $1(\mathrm{R})$ & $1(R)$ & $1(\mathrm{R})$ & $1(R)$ & $1(R)$ & $1(R)$ & $4(M)$ & $4(M)$ & $1(\mathrm{R})$ \\
\hline $\begin{array}{l}\text { 22. Assessment of volume } \\
\text { status in a critically ill } \\
\text { patient }\end{array}$ & 7 (A) & $2(R)$ & $1(\mathrm{R})$ & $1(R)$ & $1(\mathrm{R})$ & $1(\mathrm{R})$ & $1(\mathrm{R})$ & $1(\mathrm{R})$ & $1(R)$ & $1(\mathrm{R})$ & $1(\mathrm{R})$ \\
\hline \multicolumn{12}{|l|}{ Hypertensive Heart Disease } \\
\hline $\begin{array}{l}\text { 23. Initial evaluation of } \\
\text { suspected hypertensive } \\
\text { heart disease }\end{array}$ & $8(A)$ & $2(R)$ & $2(R)$ & $3(R)$ & $1(\mathrm{R})$ & $1(\mathrm{R})$ & $1(\mathrm{R})$ & $3(R)$ & $2(R)$ & $1(\mathrm{R})$ & $1(\mathrm{R})$ \\
\hline $\begin{array}{l}\text { 24. Routine evaluation of } \\
\text { systemic hypertension } \\
\text { without symptoms or } \\
\text { signs of hypertensive } \\
\text { heart disease }\end{array}$ & $5(M)$ & $1(\mathrm{R})$ & $1(\mathrm{R})$ & $1(R)$ & $1(R)$ & $1(R)$ & $1(R)$ & $1(R)$ & $1(\mathrm{R})$ & $1(\mathrm{R})$ & $1(\mathrm{R})$ \\
\hline
\end{tabular}

(Continued) 
TABLE 2. Continued

\begin{tabular}{|c|c|c|c|c|c|c|c|c|c|c|c|}
\hline Indication & $\begin{array}{l}\text { TTE (With or } \\
\text { Without 3D; } \\
\text { With Contrast as } \\
\text { Needed) }\end{array}$ & $\begin{array}{c}\text { TEE } \\
\text { (With or } \\
\text { Without 3D) }\end{array}$ & $\begin{array}{l}\text { Stress } \\
\text { Echo* }\end{array}$ & $\begin{array}{l}\text { Strain/Strain } \\
\text { Rate Imaging } \\
\text { by Speckle or } \\
\text { Tissue Doppler }\end{array}$ & $\begin{array}{l}\text { F-18 FDG } \\
\text { PET }\end{array}$ & $\begin{array}{l}\text { TC- } \\
99 m \\
\text { PYP }\end{array}$ & $\begin{array}{c}\text { MPI } \\
\text { (SPECT/ } \\
\text { PET) }\end{array}$ & CMR & CT $\dagger$ & ANG & RVG \\
\hline \multicolumn{12}{|l|}{ ACS } \\
\hline $\begin{array}{l}\text { 25. Evaluation of LV function } \\
\text { during initial presentation } \\
\text { with acute coronary } \\
\text { syndrome }\end{array}$ & 8 (A) & $2(\mathrm{R})$ & $1(\mathrm{R})$ & $1(\mathrm{R})$ & $1(\mathrm{R})$ & $1(\mathrm{R})$ & $1(\mathrm{R})$ & $3(\mathrm{R})$ & $1(\mathrm{R})$ & 7 (A) & 1 (R) \\
\hline $\begin{array}{l}\text { 26. Suspected complication of } \\
\text { myocardial ischemia/ } \\
\text { infarction, including but } \\
\text { not limited to acute mitral } \\
\text { regurgitation, ventricular } \\
\text { septal defect, free-wall } \\
\text { rupture/tamponade, } \\
\text { shock, right ventricular } \\
\text { involvement, HF, or } \\
\text { intraventricular thrombus }\end{array}$ & $9(A)$ & 7 (A) & $1(\mathrm{R})$ & 1 (R) & $1(\mathrm{R})$ & $1(\mathrm{R})$ & $1(\mathrm{R})$ & $6(M)$ & $6(M)$ & $6(\mathrm{M})$ & $2(\mathrm{R})$ \\
\hline \multicolumn{12}{|c|}{ Respiratory Failure/Exertional Shortness of Breath } \\
\hline $\begin{array}{l}\text { 27. Exertional shortness of } \\
\text { breath/dyspnea or } \\
\text { hypoxemia of uncertain } \\
\text { etiology }\end{array}$ & $8(A)$ & $3(R)$ & 7 (A) & $4(M)$ & $1(\mathrm{R})$ & $1(\mathrm{R})$ & $6(M)$ & $5(M)$ & $5(M)$ & $3(\mathrm{R})$ & 3 (R) \\
\hline $\begin{array}{l}\text { 28. Exertional shortness of } \\
\text { breath /dyspnea or } \\
\text { hypoxemia when a non- } \\
\text { cardiac etiology of } \\
\text { dyspnea has been } \\
\text { established }\end{array}$ & $4(M)$ & $1(\mathrm{R})$ & $1(\mathrm{R})$ & $1(\mathrm{R})$ & 1 (R) & $1(\mathrm{R})$ & $1(R)$ & $1(\mathrm{R})$ & $2(\mathrm{R})$ & $1(\mathrm{R})$ & $1(R)$ \\
\hline \multicolumn{12}{|l|}{ Heart Failure/Cardiomyopathy } \\
\hline $\begin{array}{l}\text { 29. Initial evaluation of known } \\
\text { or suspected HF (systolic } \\
\text { or diastolic) based on } \\
\text { symptoms, signs, or } \\
\text { abnormal test results to } \\
\text { assess systolic or diastolic } \\
\text { function and to assess for } \\
\text { possible etiology (CAD, } \\
\text { valvular disease) }\end{array}$ & $9(A)$ & $4(M)$ & 7 (A) & $6(\mathrm{M})$ & $3(\mathrm{R})$ & $1(\mathrm{R})$ & 7 (A) & 7 (A) & $6(M)$ & $6(\mathrm{M})$ & $4(M)$ \\
\hline $\begin{array}{l}\text { 30. Suspected inherited or } \\
\text { acquired cardiomyopathy } \\
\text { (e.g., restrictive, } \\
\text { infiltrative, dilated, } \\
\text { hypertrophic) }\end{array}$ & $9(A)$ & $3(\mathrm{R})$ & $3(\mathrm{R})$ & $6(M)$ & $4(M)$ & $4(M)$ & $2(R)$ & 7 (A) & $4(M)$ & $3(\mathrm{R})$ & $4(M)$ \\
\hline $\begin{array}{l}\text { 31. Evaluation of LV function } \\
\text { in patients who are } \\
\text { scheduled for or who have } \\
\text { received chemotherapy }\end{array}$ & $9(A)$ & $1(\mathrm{R})$ & $2(\mathrm{R})$ & $6(M)$ & $1(\mathrm{R})$ & $1(\mathrm{R})$ & $1(\mathrm{R})$ & $6(M)$ & $4(M)$ & $1(\mathrm{R})$ & 7 (A) \\
\hline \multicolumn{12}{|l|}{ Pulmonary Hypertension } \\
\hline $\begin{array}{l}\text { 32. Evaluation of suspected } \\
\text { pulmonary hypertension } \\
\text { including evaluation of } \\
\text { right ventricular function } \\
\text { and estimated pulmonary } \\
\text { artery pressure }\end{array}$ & 9 (A) & $3(R)$ & $3(\mathrm{R})$ & $3(\mathrm{R})$ & $1(\mathrm{R})$ & $1(\mathrm{R})$ & $1(\mathrm{R})$ & 6 (M) & $5(\mathrm{M})$ & $3(\mathrm{R})$ & $3(\mathrm{R})$ \\
\hline
\end{tabular}

(Continued) 
TABLE 2. Continued

\begin{tabular}{|c|c|c|c|c|c|c|c|c|c|c|c|}
\hline Indication & $\begin{array}{l}\text { TTE (With or } \\
\text { Without 3D; } \\
\text { With Contrast as } \\
\text { Needed) }\end{array}$ & $\begin{array}{c}\text { TEE } \\
\text { (With or } \\
\text { Without 3D) }\end{array}$ & $\begin{array}{l}\text { Stress } \\
\text { Echo* }\end{array}$ & $\begin{array}{l}\text { Strain/Strain } \\
\text { Rate Imaging } \\
\text { by Speckle or } \\
\text { Tissue Doppler }\end{array}$ & $\begin{array}{l}\text { F-18 FDG } \\
\text { PET }\end{array}$ & $\begin{array}{l}\text { Tc- } \\
99 \mathrm{~m} \\
\text { PYP }\end{array}$ & $\begin{array}{l}\text { MPI } \\
\text { (SPECT/ } \\
\text { PET) }\end{array}$ & CMR & $\mathbf{C T}+$ & ANG & RVG \\
\hline \multicolumn{12}{|l|}{ Device Therapy } \\
\hline $\begin{array}{l}\text { 33. Evaluation after } \\
\text { appropriate time interval } \\
\text { following } \\
\text { revascularization and/or } \\
\text { optimal medical therapy } \\
\text { to determine candidacy for } \\
\text { ICD/CRT and/or to } \\
\text { determine optimal choice } \\
\text { of device }\end{array}$ & $9(\mathrm{~A})$ & $2(R)$ & $1(R)$ & $2(R)$ & $1(R)$ & $1(\mathrm{R})$ & $1(R)$ & 7 (A) & $5(M)$ & $1(R)$ & 7 (A) \\
\hline $\begin{array}{l}\text { 34. Initial evaluation for CRT } \\
\text { device optimization after } \\
\text { implantation }\end{array}$ & $7(A)$ & $1(R)$ & $1(\mathrm{R})$ & $3(\mathrm{R})$ & $1(\mathrm{R})$ & $1(R)$ & $1(R)$ & $1(R)$ & $1(R)$ & $1(R)$ & $1(\mathrm{R})$ \\
\hline $\begin{array}{l}\text { 35. Known implanted pacing/ } \\
\text { ICD/CRT device with } \\
\text { symptoms possibly due to } \\
\text { suboptimal device settings }\end{array}$ & $8(A)$ & $2(R)$ & $1(R)$ & $4(M)$ & $1(R)$ & $1(\mathrm{R})$ & $1(R)$ & $1(R)$ & $1(R)$ & $1(R)$ & $1(\mathrm{R})$ \\
\hline $\begin{array}{l}\text { 36. To determine candidacy } \\
\text { for ventricular assist } \\
\text { device }\end{array}$ & $9(\mathrm{~A})$ & $3(\mathrm{R})$ & $1(\mathrm{R})$ & $1(\mathrm{R})$ & $1(\mathrm{R})$ & $1(\mathrm{R})$ & $1(R)$ & $6(M)$ & $5(M)$ & $5(M)$ & $3(\mathrm{R})$ \\
\hline $\begin{array}{l}\text { 37. Optimization of ventricular } \\
\text { assist device settings }\end{array}$ & $8(A)$ & $4(M)$ & $1(R)$ & $1(R)$ & $1(R)$ & $1(R)$ & $1(R)$ & $1(R)$ & $1(R)$ & $1(R)$ & $1(\mathrm{R})$ \\
\hline \multicolumn{12}{|l|}{ Cardiac Transplantation } \\
\hline $\begin{array}{l}\text { 38. Monitoring for rejection or } \\
\text { coronary arteriopathy in a } \\
\text { cardiac transplant } \\
\text { recipient }\end{array}$ & $8(A)$ & $2(R)$ & $3(R)$ & $4(M)$ & $2(R)$ & $1(R)$ & $4(M)$ & $5(M)$ & $3(R)$ & $5(\mathrm{M})$ & $3(\mathrm{R})$ \\
\hline $\begin{array}{l}\text { 39. Cardiac structure and } \\
\text { function evaluation in a } \\
\text { potential heart donor }\end{array}$ & $9(A)$ & $4(\mathrm{M})$ & $1(\mathrm{R})$ & $1(R)$ & $1(R)$ & $1(\mathrm{R})$ & $1(R)$ & $2(R)$ & $3(R)$ & $6(M)$ & $2(R)$ \\
\hline \multicolumn{12}{|l|}{ Other } \\
\hline $\begin{array}{l}\text { 40. Suspected pericardial } \\
\text { diseases }\end{array}$ & $9(\mathrm{~A})$ & $4(\mathrm{M})$ & $1(\mathrm{R})$ & $5(\mathrm{M})$ & $1(R)$ & 1 (R) & $1(\mathrm{R})$ & 7 (A) & $7(\mathrm{~A})$ & $1(R)$ & $1(\mathrm{R})$ \\
\hline $\begin{array}{l}\text { 41. Initial evaluation of cardiac } \\
\text { mass, suspected tumor or } \\
\text { thrombus, or potential } \\
\text { cardiac source of emboli }\end{array}$ & $9(\mathrm{~A})$ & $7(A)$ & $1(\mathrm{R})$ & 1 (R) & $1(\mathrm{R})$ & 1 (R) & $1(R)$ & $7(\mathrm{~A})$ & $7(\mathrm{~A})$ & 1 (R) & 1 (R) \\
\hline $\begin{array}{l}\text { 42. Suspected acute aortic } \\
\text { pathology including acute } \\
\text { aortic syndrome }\end{array}$ & 7 (A) & $8(A)$ & $1(R)$ & 1 (R) & $1(R)$ & 1 (R) & $1(R)$ & $8(A)$ & $9(A)$ & $3(R)$ & $1(\mathrm{R})$ \\
\hline
\end{tabular}

TTE, Transthoracic echocardiography; 3D, 3-dimensional; TEE, transesophageal echocardiography; F-18 FDG, fluorodeoxyglucose F18; PET, positron emission tomography; TC-99m PYP, technetium-99m pyrophosphate; MPI, myocardial perfusion imaging; SPECT, single-photon emission computed tomography; $C M R$, cardiovascular magnetic resonance imaging; $C T$, computed tomography; $A N G$, angiography/ventriculography/aortography; $R V G$, radionuclide ventriculography; $A$, appropriate; $R$, rarely appropriate; $M$, may be appropriate; $L B B B$, left bundle branch block; $R B B B$, right bundle branch block; $V P C$, ventricular premature contraction; $V T$, ventricular tachycardia; $V F$, ventricular fibrillation; $S V T$, supraventricular tachycardia; $H F$, heart failure; $A C S$, acute coronary syndrome; $L V$, left ventricular; $C A D$, coronary artery disease; $I C D$, implantable cardioverterdefibrillator; $C R T$, cardiac resynchronization therapy. *Stress echo comprises exercise stress echocardiography and dobutamine stress echocardiography. $\dagger$ Could include $C T$ angiography of the coronaries or any other vessel. 


\subsection{Evaluation of Cardiac Structure and Function in Patients Who Had Prior Testing}

TABLE 3. Sequential or follow-up testing to clarify initial diagnostic testing

\begin{tabular}{|c|c|c|c|c|c|c|c|c|c|c|}
\hline & $\begin{array}{l}\text { TTE (With or } \\
\text { Without 3D; } \\
\text { With Contrast } \\
\text { as Needed) }\end{array}$ & $\begin{array}{c}\text { TEE } \\
\text { (With or } \\
\text { Without 3D }\end{array}$ & $\begin{array}{l}\text { Strain/Strain } \\
\text { Rate Imaging } \\
\text { by Speckle or } \\
\text { Tissue Doppler }\end{array}$ & $\begin{array}{l}\text { Exercise } \\
\text { SE/DSE }\end{array}$ & $\begin{array}{l}\text { F-18 } \\
\text { FDG- } \\
\text { PET }\end{array}$ & $\begin{array}{l}\text { Tc- } \\
99 m \\
\text { PYP }\end{array}$ & $\begin{array}{l}\text { MPI } \\
\text { (SPECT/ } \\
\text { PET) }\end{array}$ & CMR & CT & ANG \\
\hline $\begin{array}{l}\text { 43. Left ventricular systolic dysfunction in } \\
\text { the absence of severe valvular disease }\end{array}$ & & $3(\mathrm{R})$ & $3(\mathrm{R})$ & 7 (A) & $4(\mathrm{M})$ & $3(\mathrm{R})$ & 7 (A) & 7 (A) & 7 (A) & 7 (A) \\
\hline $\begin{array}{l}\text { 44. Pulmonary hypertension in the absence } \\
\text { of severe valvular disease }\end{array}$ & & $6(\mathrm{M})$ & $4(M)$ & $4(\mathrm{M})$ & 1 (R) & $1(\mathrm{R})$ & 1 (R) & 7 (A) & 7 (A) & $3(\mathrm{R})$ \\
\hline $\begin{array}{l}\text { 45. Excluding CAD in patients with } \mathrm{HF} \text { and } \\
\text { LV systolic dysfunction without angina }\end{array}$ & & 1 (R) & 1 (R) & 7 (A) & $3(\mathrm{R})$ & $1(\mathrm{R})$ & 7 (A) & 7 (A) & 7 (A) & $8(\mathrm{~A})$ \\
\hline $\begin{array}{l}\text { 46. New or increasing HF symptoms } \\
\text { despite adherence to medical therapy }\end{array}$ & & $4(\mathrm{M})$ & $3(\mathrm{R})$ & $6(\mathrm{M})$ & $3(\mathrm{R})$ & 2 (R) & $6(\mathrm{M})$ & $6(\mathrm{M})$ & 5 (M) & $6(M)$ \\
\hline $\begin{array}{l}\text { 47. Comprehensive further evaluation of } \\
\text { undefined cardiomyopathy }\end{array}$ & & $3(\mathrm{R})$ & $5(\mathrm{M})$ & $5(\mathrm{M})$ & 5 (M) & $4(\mathrm{M})$ & $6(\mathrm{M})$ & 8 (A) & 6 (M) & 7 (A) \\
\hline $\begin{array}{l}\text { 48. Evaluation of suspected cardiac } \\
\text { sarcoidosis }\end{array}$ & & $2(\mathrm{R})$ & $3(\mathrm{R})$ & 1 (R) & 7 (A) & $2(\mathrm{R})$ & 3 (R) & 8 (A) & $3(\mathrm{R})$ & $1(\mathrm{R})$ \\
\hline $\begin{array}{l}\text { 49. Evaluation of suspected cardiac } \\
\text { amyloidosis }\end{array}$ & & 1 (R) & $6(\mathrm{M})$ & 1 (R) & $2(\mathrm{R})$ & 7 (A) & $2(\mathrm{R})$ & 8 (A) & $2(\mathrm{R})$ & $1(\mathrm{R})$ \\
\hline $\begin{array}{l}\text { 50. Evaluation of suspected hypertrophic } \\
\text { cardiomyopathy }\end{array}$ & & $4(\mathrm{M})$ & 7 (A) & 7 (A) & 1 (R) & $1(\mathrm{R})$ & 1 (R) & 8 (A) & $5(M)$ & $2(\mathrm{R})$ \\
\hline $\begin{array}{l}\text { 51. Further characterization of a known or } \\
\text { suspected, incidentally noted, small } \\
\text { cardiovascular implantable electronic } \\
\text { device-related thrombus identified by } \\
\text { TTE in an asymptomatic patient }\end{array}$ & & $2(R)$ & $1(\mathrm{R})$ & 1 (R) & 1 (R) & $1(\mathrm{R})$ & 1 (R) & $1(\mathrm{R})$ & $1(\mathrm{R})$ & $1(\mathrm{R})$ \\
\hline $\begin{array}{l}\text { 52. Comprehensive further evaluation of } \\
\text { dilated aortic sinuses or ascending aorta } \\
\text { identified by TTE }\end{array}$ & & 7 (A) & 1 (R) & 1 (R) & 1 (R) & $1(\mathrm{R})$ & 1 (R) & 8 (A) & $8(A)$ & $3(\mathrm{R})$ \\
\hline $\begin{array}{l}\text { 53. Evaluation of the aortic sinuses, } \\
\text { sinotubular junction, or ascending aorta } \\
\text { in patients with bicuspid aortic valve } \\
\text { when morphology cannot be assessed } \\
\text { accurately or fully by TTE }\end{array}$ & & 7 (A) & 1 (R) & 1 (R) & 1 (R) & $1(\mathrm{R})$ & 1 (R) & $8(A)$ & $8(\mathrm{~A})$ & $3(\mathrm{R})$ \\
\hline $\begin{array}{l}\text { 54. Further anatomic characterization of } \\
\text { anomalous coronary arteries identified } \\
\text { by invasive coronary angiography }\end{array}$ & $2(\mathrm{R})$ & 1 (R) & 1 (R) & 1 (R) & 1 (R) & $1(\mathrm{R})$ & 1 (R) & 7 (A) & 9 (A) & \\
\hline
\end{tabular}

TTE, Transthoracic echocardiography; 3D, 3-dimensional; TEE, transesophageal echocardiography; $S E$, stress echocardiography; $D S E$, dobutamine stress echocardiography; F-18 FDG, fluorodeoxyglucose F18; PET, positron emission tomography; Tc-99m PYP, technetium-99m pyrophosphate; $M P I$, myocardial perfusion imaging; SPECT, singlephoton emission computed tomography; $C M R$, cardiovascular magnetic resonance imaging; $C T$, computed tomography; $A N G$, angiography/ventriculography/aortography; $R$, rarely appropriate; $A$, appropriate; $M$, may be appropriate; $C A D$, coronary artery disease; $H F$, heart failure; $A U C$, Appropriate Use Criteria. *For more specific scenarios, please refer to the Heart Failure AUC. †Modalities grayed out assumes modality has been performed. 
TABLE 4. Sequential or follow-up testing: asymptomatic or stable symptoms

\begin{tabular}{|c|c|c|c|c|c|c|c|c|}
\hline Indication & $\begin{array}{l}\text { TTE (With or } \\
\text { Without 3D; } \\
\text { With Contrast } \\
\text { as Needed) }\end{array}$ & $\begin{array}{c}\text { TEE } \\
\text { (With or } \\
\text { Without 3D) }\end{array}$ & $\begin{array}{l}\text { Strain/Strain } \\
\text { Rate Imaging } \\
\text { by Speckle } \\
\text { or Tissue } \\
\text { Doppler }\end{array}$ & $\begin{array}{l}\text { F-18 } \\
\text { FDG-PET }\end{array}$ & $\begin{array}{c}\text { Tc-99m } \\
\text { PYP }\end{array}$ & CMR & CT & RVG \\
\hline $\begin{array}{l}\text { 55. Re-evaluation }(<1 \mathrm{y}) \text { in a patient at risk for HF } \\
\text { without structural heart disease on prior TTE } \\
\text { and no change in clinical status or cardiac } \\
\text { examination (stage } \mathrm{A} \text { ) }\end{array}$ & 2 (R) & 1 (R) & $1(R)$ & $1(R)$ & 1 (R) & $1(\mathrm{R})$ & 1 (R) & $1(R)$ \\
\hline $\begin{array}{l}\text { 56. Re-evaluation of known hypertensive heart } \\
\text { disease without a change in clinical status or } \\
\text { cardiac examination (stage } A)(<1 \mathrm{y})\end{array}$ & 2 (R) & 1 (R) & 1 (R) & 1 (R) & 1 (R) & 1 (R) & 1 (R) & $1(\mathrm{R})$ \\
\hline $\begin{array}{l}\text { 57. Re-evaluation ( }<1 \mathrm{y} \text { ) of HF (systolic or diastolic) } \\
\text { cardiomyopathy or HF without a change in } \\
\text { clinical status or cardiac examination }\end{array}$ & $2(R)$ & $1(\mathrm{R})$ & 1 (R) & $1(\mathrm{R})$ & $1(\mathrm{R})$ & 1 (R) & 1 (R) & $1(R)$ \\
\hline $\begin{array}{l}\text { 58. Re-evaluation }(<1 \mathrm{y}) \text { in a patient previously or } \\
\text { currently undergoing therapy with potentially } \\
\text { cardiotoxic agents }\end{array}$ & 7 (A) & 1 (R) & 7 (A) & $1(R)$ & 1 (R) & $5(\mathrm{M})$ & $2(R)$ & 7 (A) \\
\hline $\begin{array}{l}\text { 59. Re-evaluation }(<1 \text { y) of known aortic dilatation } \\
\text { at baseline study to assess changes in rate of } \\
\text { expansion or size in patient without bicuspid } \\
\text { aortic valve }\end{array}$ & $3(R)$ & 1 (R) & 1 (R) & $1(R)$ & 1 (R) & $2(R)$ & $3(\mathrm{R})$ & $1(R)$ \\
\hline $\begin{array}{l}\text { 60. Re-evaluation }(<1 \mathrm{y}) \text { of the size and } \\
\text { morphology of the aortic sinuses and ascending } \\
\text { aorta in patients with a bicuspid aortic valve and } \\
\text { an aortic diameter }>4 \mathrm{~cm} \text { without characteristics } \\
\text { mentioned in } \# 61\end{array}$ & $2(R)$ & 1 (R) & 1 (R) & 1 (R) & 1 (R) & 3 (R) & 3 (R) & $1(R)$ \\
\hline $\begin{array}{l}\text { 61. Re-evaluation ( }<1 \mathrm{y} \text { ) of the size and morphology } \\
\text { of the aortic sinuses and ascending aorta in } \\
\text { patients with a bicuspid aortic valve and an } \\
\text { aortic diameter }>4 \mathrm{~cm} \text { with one of the } \\
\text { following: } \\
\text { - Aortic dilatation }>4.5 \mathrm{~cm} \\
\text { - Rapid rate of change in aortic diameter } \\
\text { - Family history of aortic dissection }\end{array}$ & 3 (R) & 1 (R) & 1 (R) & 1 (R) & 1 (R) & $5(M)$ & $5(M)$ & 1 (R) \\
\hline $\begin{array}{l}\text { 62. Re-evaluation }(<1 \text { y) of known moderate or } \\
\text { greater pulmonary hypertension without change } \\
\text { in clinical status or cardiac examination }\end{array}$ & $4(M)$ & 1 (R) & 1 (R) & $1(R)$ & $1(\mathrm{R})$ & 1 (R) & 1 (R) & 1 (R) \\
\hline $\begin{array}{l}\text { 63. Re-evaluation }(\geq 1 \mathrm{y}) \text { of known moderate or } \\
\text { greater pulmonary hypertension without change } \\
\text { in clinical status or cardiac examination }\end{array}$ & 7 (A) & 1 (R) & $1(\mathrm{R})$ & $1(R)$ & $1(\mathrm{R})$ & $2(R)$ & 1 (R) & 1 (R) \\
\hline $\begin{array}{l}\text { 64. Re-evaluation of chronic asymptomatic } \\
\text { pericardial effusion when findings would } \\
\text { potentially alter therapy }\end{array}$ & $7(A)$ & $2(R)$ & 1 (R) & $1(R)$ & $1(R)$ & $4(M)$ & $3(R)$ & $1(R)$ \\
\hline $\begin{array}{l}\text { 65. Further clarification of suspected pericardial } \\
\text { constriction when findings of TTE including } \\
\text { tissue Doppler is unclear }\end{array}$ & $1(R)$ & $3(\mathrm{R})$ & $1(\mathrm{R})$ & 1 (R) & $1(\mathrm{R})$ & 7 (A) & 7 (A) & 1 (R) \\
\hline $\begin{array}{l}\text { 66. Re-evaluation of intracardiac mass when } \\
\text { findings would potentially alter therapy }\end{array}$ & $8(A)$ & 7 (A) & $1(\mathrm{R})$ & $1(\mathrm{R})$ & $1(\mathrm{R})$ & $8(A)$ & 8 (A) & 1 (R) \\
\hline $\begin{array}{l}\text { 67. Re-evaluation of prior TEE findings for interval } \\
\text { change (e.g., resolution of atrial thrombus after } \\
\text { anticoagulation) when no change in therapy is } \\
\text { anticipated. }\end{array}$ & 1 (R) & 1 (R) & $1(\mathrm{R})$ & $1(R)$ & $1(R)$ & $1(R)$ & $1(R)$ & 1 (R) \\
\hline
\end{tabular}

TTE, Transthoracic echocardiography; $3 D$, 3-dimensional; TEE, transesophageal echocardiography; $F$ - 18 FDG, fluorodeoxyglucose F18; PET, positron emission tomography; $T c-99 m$ PYP, technetium-99m pyrophosphate; $C M R$, cardiovascular magnetic resonance imaging; $C T$, computed tomography; $R V G$, radionuclide ventriculography; $H F$, heart failure; $R$, rarely appropriate; $A$, appropriate; $M$, may be appropriate. 
TABLE 5. Sequential or follow-up testing: new or worsening symptoms or to guide therapy

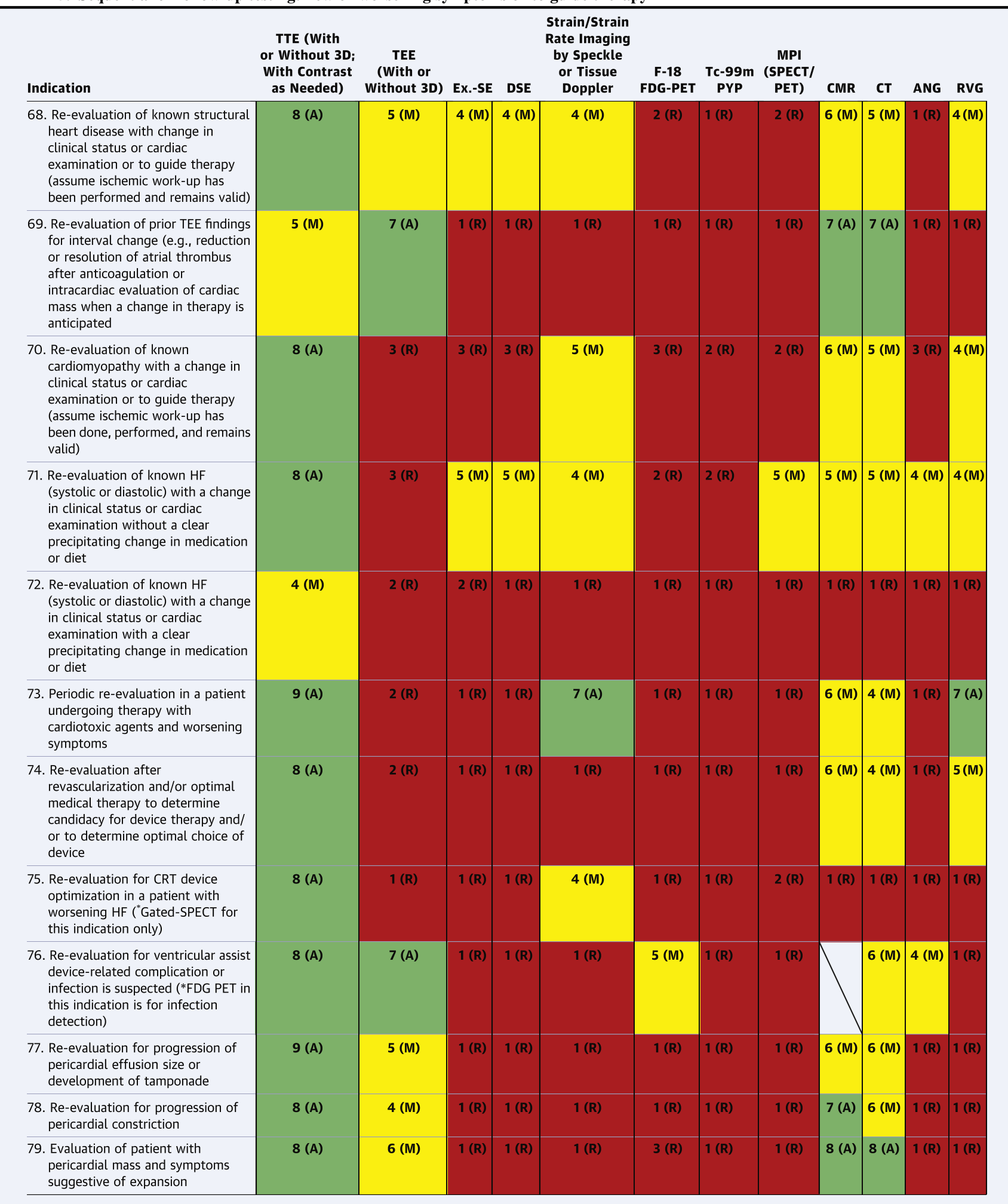

(Continued) 
TABLE 5. Continued

\begin{tabular}{|c|c|c|c|c|c|c|c|c|c|c|c|c|}
\hline Indication & $\begin{array}{l}\text { TTE (With } \\
\text { or Without 3D; } \\
\text { With Contrast } \\
\text { as Needed) }\end{array}$ & $\begin{array}{c}\text { TEE } \\
\text { (With or } \\
\text { Without 3D) }\end{array}$ & Ex.-SE & DSE & $\begin{array}{l}\text { Strain/Strain } \\
\text { Rate Imaging } \\
\text { by Speckle } \\
\text { or Tissue } \\
\text { Doppler }\end{array}$ & $\begin{array}{c}\text { F-18 } \\
\text { FDG-PET }\end{array}$ & $\begin{array}{c}\text { Tc-99m } \\
\text { PYP }\end{array}$ & $\begin{array}{c}\text { MPI } \\
\text { (SPECT/ } \\
\text { PET) }\end{array}$ & CMR & Ст & ANG & RVG \\
\hline $\begin{array}{l}\text { 80. Re-evaluation of known ascending } \\
\text { aortic dilatation or history of aortic } \\
\text { dissection with a change in clinical } \\
\text { status (excluding acute coronary } \\
\text { syndrome) or cardiac examination } \\
\text { or when findings may alter } \\
\text { management or therapy }\end{array}$ & $8(\mathrm{~A})$ & 7 (A) & 1 (R) & $1(R)$ & 1 (R) & 1 (R) & 1 (R) & 1 (R) & 8 (A) & $8(A)$ & $2(R)$ & $1(R)$ \\
\hline $\begin{array}{l}\text { 81. Re-evaluation of known pulmonary } \\
\text { hypertension with change in } \\
\text { clinical status or cardiac } \\
\text { examination or to guide therapy }\end{array}$ & 8 (A) & 4 (M) & $4(M)$ & $1(R)$ & 1 (R) & 1 (R) & $1(R)$ & 1 (R) & $5(M)$ & $6(\mathrm{M})$ & $3(\mathrm{R})$ & $1(\mathrm{R})$ \\
\hline
\end{tabular}

TTE, Transthoracic echocardiography; 3D, 3-dimensional; TEE, transesophageal echocardiography; Ex.-SE, exercise stress echocardiography; $D S E$, dobutamine stress echocardiography; F-18 FDG, fluorodeoxyglucose F18; PET, positron emission tomography; $T c-99 \mathrm{~m} P Y P$, technetium-99m pyrophosphate; $M P I$, myocardial perfusion imaging; $S P E C T$, single-photon emission computed tomography; $C M R$, cardiovascular magnetic resonance imaging; $C T$, computed tomography; $A N G$, angiography/ventriculography/aortography; $R V G$, radionuclide ventriculography; $A$, appropriate; $M$, may be appropriate; $R$, rarely appropriate; $H F$, heart failure; $C R T$, cardiac resynchronization therapy.

\subsection{Evaluation of Cardiac Structure and Function in Patients Undergoing Transcatheter Intervention for Structural Heart Disease}

TABLE 6. Imaging for the evaluation of TIA or ischemic stroke

\begin{tabular}{|c|c|c|c|c|c|c|c|c|c|}
\hline & $\begin{array}{c}\text { TTE (with } \\
\text { agitated saline } \\
\text { injection; with or } \\
\text { without 3D; with } \\
\text { contrast as } \\
\text { needed) }\end{array}$ & $\begin{array}{c}\text { TEE (with } \\
\text { agitated saline } \\
\text { injection; } \\
\text { with or without 3D) }\end{array}$ & TCD & $\begin{array}{l}\text { MRA } \\
\text { H/N }\end{array}$ & $\begin{array}{l}\text { CTA } \\
\text { H/N }\end{array}$ & $\begin{array}{c}\text { Cardiac } \\
\text { MR }\end{array}$ & $\begin{array}{c}\text { Cardiac } \\
\text { CT }\end{array}$ & ANG & $\begin{array}{l}\text { Carotid } \\
\text { Doppler }\end{array}$ \\
\hline $\begin{array}{l}\text { 82. Initial evaluation of patient to exclude } \\
\text { cardiac origin of TIA or ischemic stroke: } \\
\text { Intracardiac masses (thrombus, } \\
\text { vegetation) } \\
\text { - Valvular pathology }\end{array}$ & $8(A)$ & 7 (A) & $6(M)$ & $6(\mathrm{M})$ & $6(M)$ & $5(\mathrm{M})$ & $5(M)$ & $3(R)$ & 7 (A) \\
\hline 83. Assessment of intracranial arteries & & & $6(M)$ & $8(A)$ & 8 (A) & & & $5(M)$ & \\
\hline $\begin{array}{l}\text { 84. Assessment of extracranial arteries } \\
\text { (evaluation of the carotid and vertebral } \\
\text { arteries) }\end{array}$ & & & & $8(A)$ & $8(A)$ & & & $5(M)$ & $8(A)$ \\
\hline $\begin{array}{l}\text { 85. Provocative maneuvers (Valsalva, } \\
\text { cough) to assess for the presence of: } \\
\text { Right-to-left intracardiac shunt }\end{array}$ & $8(A)$ & $7(A)$ & 7 (A) & $1(R)$ & $1(R)$ & $1(R)$ & $1(R)$ & $1(R)$ & $1(R)$ \\
\hline
\end{tabular}

TTE, Transthoracic echocardiography; 3D, 3-dimensional; TEE, transesophageal echocardiography; TCD, transcranial Doppler; $M R A$, magnetic resonance angiography; $H / N$, head and neck; $C T A$, computed tomography angiography; $M R$, magnetic resonance; $C T$, computed tomography; $A N G$, angiography/ventriculography/aortography; $T I A$, transient ischemic attack; $A$, appropriate; $M$, may be appropriate; $R$, rarely appropriate. 


\subsubsection{Imaging for the evaluation of patent foramen ovale or atrial septal defect}

TABLE 7. Preprocedural evaluation for closure of PFO or atrial septal defect

\begin{tabular}{|c|c|c|c|c|c|c|}
\hline Indication & $\begin{array}{l}\text { TTE (With Agitated } \\
\text { Saline Injection; With } \\
\text { or Without 3D; With } \\
\text { Contrast as Needed) }\end{array}$ & $\begin{array}{c}\text { TEE (With } \\
\text { Agitated Saline } \\
\text { Injection; With } \\
\text { or Without 3D) }\end{array}$ & 3D-TEE & CMR & CTA & ANG \\
\hline $\begin{array}{l}\text { 86. Preprocedure assessment for PFO: } \\
\text { - Atrial appendage thrombus } \\
\text { - Spontaneous echo contrast (slow blood flow) } \\
\text { - Aortic atheroma } \\
\text { - Cardiac masses } \\
\text { - Vegetations }\end{array}$ & 7 (A) & $8(A)$ & $5(\mathrm{M})$ & $1(\mathrm{R})$ & $1(\mathrm{R})$ & 1 (R) \\
\hline $\begin{array}{l}\text { 87. Preprocedure assessment for: } \\
\text { - Atrial septum anatomy } \\
\text { - Atrial septum aneurysm } \\
\text { - Suitability for percutaneous device closure }\end{array}$ & 7 (A) & $8(A)$ & $8(A)$ & 7 (A) & $7(A)$ & $1(\mathrm{R})$ \\
\hline
\end{tabular}

TTE, Transthoracic echocardiography; 3D, 3-dimensional; TEE, transesophageal echocardiography; $C M R$, cardiovascular magnetic resonance imaging; $C T A$, computed tomography angiography; $A N G$, angiography/ventriculography/aortography; $P F O$, patent foramen ovale; $A$, appropriate; $M$, may be appropriate; $R$, rarely appropriate.

TABLE 8. Intra-procedural guidance for closure of PFO or ASD

\begin{tabular}{|c|c|c|c|c|c|c|}
\hline Indication & $\begin{array}{l}\text { TTE (With Agitated } \\
\text { Saline Injection; With } \\
\text { or Without 3D; With } \\
\text { Contrast as Needed) }\end{array}$ & $\begin{array}{l}\text { TEE (With Agitated } \\
\text { Saline Injection) }\end{array}$ & $\begin{array}{l}\text { 3D-TEE (With } \\
\text { Agitated Saline } \\
\text { Injection) }\end{array}$ & $\begin{array}{l}\text { ICE (With or } \\
\text { Without 3D) }\end{array}$ & TCD & Fluoro \\
\hline $\begin{array}{l}\text { 88. Intraprocedural guidance in patient with either: } \\
\text { - ASD of simple anatomy } \\
\text { - No aneurysmal atrial septum } \\
\text { - PFO with short tunnel }\end{array}$ & $3(R)$ & $7(A)$ & $7(A)$ & $8(A)$ & 1 (R) & $7(A)$ \\
\hline $\begin{array}{l}\text { 89. Intraprocedural guidance in patient with either: } \\
\text { - ASD with complex anatomy } \\
\text { - Aneurysmal interatrial septum } \\
\text { - PFO with long tunnel }\end{array}$ & $3(R)$ & $8(A)$ & $7(A)$ & $8(A)$ & $1(\mathrm{R})$ & 7 (A) \\
\hline
\end{tabular}

TTE, Transthoracic echocardiography; 3D, 3-dimensional; TEE, transesophageal echocardiography; ICE, intracardiac echocardiography; TCD, transcranial Doppler; Fluoro, fluoroscopy; $A S D$, atrial septal defect; $P F O$, patent foramen ovale; $R$, rarely appropriate; $A$, appropriate.

TABLE 9. Assessment following closure of PFO or ASD

\begin{tabular}{|c|c|c|c|c|}
\hline Indication & $\begin{array}{l}\text { TTE (With Agitated } \\
\text { Saline Injection; With } \\
\text { or Without 3D; With } \\
\text { Contrast as Needed) }\end{array}$ & $\begin{array}{l}\text { TEE (With or } \\
\text { Without 3D Agitated } \\
\text { Saline Injection) }\end{array}$ & CMR & Ст \\
\hline $\begin{array}{l}\text { 90. 6-month routine scheduled follow-up ASD/PFO device } \\
\text { closure for position of device and integrity of device } \\
\text { - PFO patency } \\
\text { - Thrombus formation }\end{array}$ & 7 (A) & $4(\mathrm{M})$ & $3(\mathrm{R})$ & $2(R)$ \\
\hline $\begin{array}{l}\text { 91. Nonroutine follow up of ASD/PFO device closure and clinical } \\
\text { concern for infection, malposition, embolization or persistent } \\
\text { shunt. }\end{array}$ & 8 (A) & 8 (A) & $4(\mathrm{M})$ & $4(\mathrm{M})$ \\
\hline
\end{tabular}

TTE, Transthoracic echocardiography; $3 D$, 3-dimensional; TEE, transesophageal echocardiography; $C M R$, cardiac magnetic resonance; $C T$, computed tomography; $A S D$, atrial septal defect; $P F O$, patent foramen ovale; $A$, appropriate; $M$, may be appropriate; $R$, rarely appropriate. 


\subsubsection{Imaging for the evaluation of left atrial appendage occlusion device}

TABLE 10. Pre-procedural evaluation for LAA occlusion

\begin{tabular}{|c|c|c|c|c|}
\hline Indication & $\begin{array}{l}\text { TTE (With or Without 3D; } \\
\text { With Contrast as Needed) }\end{array}$ & TEE (With or Without 3D) & CTA & CMR \\
\hline $\begin{array}{l}\text { 92. Evaluate for: } \\
\text { All cardiac chambers } \\
\text { - LV function } \\
\text { - Interatrial septum } \\
\text { - Valve function }\end{array}$ & $8(A)$ & $8(A)$ & 7 (A) & $5(M)$ \\
\hline $\begin{array}{l}\text { 93. Evaluate for: } \\
\text { - LA/LAA thrombus } \\
\text { - Spontaneous echo contrast/slow blood flow }\end{array}$ & $5(M)$ & 9 (A) & 7 (A) & 5 (M) \\
\hline $\begin{array}{l}\text { 94. Assess: } \\
\text { - LAA morphology } \\
\text { - Baseline LAA dimensions } \\
\text { - Ostial morphology and dimension } \\
\text { - Maximum length of dominant lobe }\end{array}$ & $6(M)$ & 9 (A) & 7 (A) & $5(\mathrm{M})$ \\
\hline
\end{tabular}

TTE, Transthoracic echocardiography; 3D, 3-dimensional; TEE, transesophageal echocardiography; $C T A$, computed tomography angiography; $C M R$, cardiac magnetic resonance; $L V$, left ventricular; $A$, appropriate; $M$, may be appropriate; $L A$, left atrial; $L A A$, left atrial appendage.

TABLE 11. Intraprocedural guidance for LAA occlusion

\begin{tabular}{|c|c|c|c|c|}
\hline Indication & $\begin{array}{l}\text { TTE (With or Without 3D; } \\
\text { With Contrast as Needed) }\end{array}$ & TEE (With or Without 3D) & ICE & Fluoro \\
\hline 95. Reassess for LAA thrombus & & $9(\mathrm{~A})$ & $6(M)$ & $4(M)$ \\
\hline 96. Assess LAA size and morphology: select device size & & $9(\mathrm{~A})$ & $6(M)$ & $5(M)$ \\
\hline 97. Guide delivery and deployment of the device & & $9(\mathrm{~A})$ & $6(M)$ & $8(A)$ \\
\hline $\begin{array}{l}\text { 98. Check for: } \\
\text { Leaks around device } \\
\text { Disruption of mitral inflow } \\
\text { Disruption of pulmonary vein flow }\end{array}$ & & $9(\mathrm{~A})$ & $6(\mathrm{M})$ & 7 (A) \\
\hline 99. Assess adequacy of LAA occlusion & & $9(A)$ & $6(M)$ & 7 (A) \\
\hline 100. Screen for procedural complications & 7 (A) & $9(\mathrm{~A})$ & $7(\mathrm{~A})$ & 7 (A) \\
\hline
\end{tabular}

TTE, Transthoracic echocardiography; 3D, 3-dimensional; TEE, transesophageal echocardiography; ICE, intracardiac echo; Fluoro, fluoroscopy; LAA, left atrial appendage; $A$, appropriate; $M$, may be appropriate.

TABLE 12. Assessment following LAA occlusion

\begin{tabular}{|c|c|c|c|c|c|}
\hline & TTE (With or Without 3D) & TEE (With or Without 3D) & CTA & CMR & Fluoro \\
\hline $\begin{array}{l}\text { 101. Prior to discharge to assess: } \\
\text { - Device position } \\
\text { - Presence of pericardial effusion } \\
\text { - Presence of thrombus around the device } \\
\text { - Mitral valve function } \\
\text { - LV function }\end{array}$ & $6(M)$ & $3(\mathrm{R})$ & $2(R)$ & $1(\mathrm{R})$ & $1(\mathrm{R})$ \\
\hline $\begin{array}{l}\text { 102. Surveillance at } 45 \text { days or FDA guidance/guidelines for follow-up: } \\
\text { Assess device stability } \\
\text { Exclude migration, displacement, or erosion } \\
\text { - Assess device leak }\end{array}$ & $4(M)$ & 8 (A) & $3(R)$ & $2(R)$ & $1(\mathrm{R})$ \\
\hline 103. Long-term follow-up (assume device integrity) & $5(M)$ & $4(\mathrm{M})$ & $2(R)$ & $2(R)$ & $1(\mathrm{R})$ \\
\hline
\end{tabular}

TTE, Transthoracic echocardiography; 3D, 3-dimensional; TEE, transesophageal echocardiography; CTA, computed tomography angiography; CMR, cardiac magnetic resonance; Fluoro, fluoroscopy; $L V$, left ventricular; $M$, may be appropriate; $R$, rarely appropriate; FDA, Food and Drug Administration; $A$, appropriate. 


\section{DISCUSSION}

AUC are intended to inform clinicians, patients, and health policy makers about the reasonable use of technologies to help improve patient symptoms and health outcomes. Since 2005 , the ACC, along with its professional partners, has worked to provide criteria for both invasive and noninvasive testing and selected treatments, further expanding the AUC portfolio., ${ }^{2,3,10-14}$

The 2019 Appropriate Use Criteria for Multimodality Imaging in the Assessment of Cardiac Structure and Function in Nonvalvular Heart Disease is the culmination of the analysis of various modalities used in the evaluation and treatment of patients with nonvalvular heart disease. The document signals a shift from documents evaluating a single modality in various disease states to documents evaluating multiple imaging modalities and focusing on evidence and clinical experience within a given disease category. We believe that this approach better reflects clinical decision making in real-world scenarios and offers the diagnostic choices available to the clinician.

Because a given modality may address diverse disease states, indications previously compiled in a single document may be spread over several AUC documents. The previous nonvalvular heart disease-related indications that the current paper supplants are contained in the echocardiography (echo) ${ }^{14}$ radionuclide imaging, ${ }^{13}$ and computed tomography/magnetic resonance imaging AUC documents. ${ }^{11,12}$ Other indications in these documents remain in force until these scenarios are evaluated in subsequent documents.

The tables in this paper are organized to reflect the spectrum of patients with nonvalvular heart disease-from patients with no symptoms suspected of having nonvalvular heart disease to patients with signs and symptoms ranging from mild to severe. The first 2 tables are for initial evaluation when no prior imaging has been done. As noted, the diagnostic choices vary among the tables and reflect the options that would be considered in the initial evaluation by most clinicians. If a diagnostic test would seldom or never be considered, it was not included as an option for the rating panel.

Table 1 addresses the initial evaluation of an asymptomatic patient. ${ }^{15}$ This encompasses a variety of clinical scenarios, including the evaluation of patients with a known disease state that could be associated with structural heart disease, evaluation for structural heart disease in firstdegree relatives of a patient with inherited cardiomyopathy, ${ }^{16}$ initial evaluation prior to exposure to potentially cardiotoxic medications, ${ }^{17,18}$ and participation for asymptomatic athletes with and without a family history of heart disease. ${ }^{19}$ Imaging of the thoracic aorta is evaluated in patients with a known or suspected connective tissue or genetic condition that predisposes patients to aortic aneurysm or in patients whose relatives have a known aortic aneurysm or dissection. ${ }^{20,21}$ Finally, suspected pulmonary arterial hypertension (including the evaluation of right ventricular function) is evaluated in patients at risk for developing pulmonary hypertension. ${ }^{22}$

As might be expected, transthoracic echo is rated Appropriate in all of these scenarios. Strain rate imaging by speckle tracking is also rated May Be Appropriate for specific indications. Cardiac MR and cardiac computed tomography $(\mathrm{CT})$ are rated May Be Appropriate for specific cardiac indications and are rated Appropriate for the evaluation of the thoracic aorta.

Table 2 addresses initial evaluation of a patient with clinical signs and or symptoms of cardiac disease. ${ }^{18}$ This is further subdivided into the categories of arrhythmias or conduction disorders; palpitations/presyncope/syncope ${ }^{16,23-26}$; hypotension or hemodynamic instability; hypertensive heart disease; acute coronary syndromes; respiratory failure; heart failure; pulmonary hypertension ${ }^{27-29}$; device therapy ${ }^{30,31}$; and cardiac transplantation, including monitoring for rejection in a recipient and evaluation of structure and function in a potential heart donor. ${ }^{22,32} \mathrm{In}$ addition, evaluation for suspected pericardial disease or suspected acute aortic pathology and initial evaluation of cardiac mass or potential cardiac source of embolism are examined. As might be expected, the modality chosen depends upon the disease state for which the clinician wishes to evaluate. That being said, transthoracic echo is the modality recognized as Appropriate in most of the scenarios. Coronary angiography is also included and is found to be Appropriate in the evaluation of the patient with sustained ventricular tachycardia or ventricular fibrillation but is not an initial testing modality across other scenarios. The presence of atrial fibrillation in which ischemia may be a trigger resulted in a May $\mathrm{Be}$ Appropriate rating for single-photon emission computed tomography imaging and stress echo. Scenarios such as a newly diagnosed right bundle branch block, supraventricular tachycardia, and palpitations without other symptoms or signs of heart disease resulted in a May Be Appropriate rating for transthoracic echo and a Rarely Appropriate rating for all other modalities. Mechanical complications of myocardial infarction are evaluated with an Appropriate rating for both transthoracic and transesophageal echo, but cardiac MR and cardiac CT as well as coronary angiography with ventriculography received a May Be Appropriate rating. F-18 fluorodeoxyglucose-positron emission tomography and technetium $99 \mathrm{~m}$ pyrophosphate injection are also evaluated and received a May Be Appropriate rating for the evaluation of cardiac sarcoid and amyloid, respectively.

Section 6.2 evaluates cardiac structure and function in patients who have undergone prior testing. As examined 
in Table 3, this sequential testing is done to clarify the initial diagnosis. These are instances in which the initial imaging modality - commonly transthoracic echo-has not yielded a definitive diagnosis. Scenarios included here are left ventricular dysfunction not explained by the presence of severe valvular disease, pulmonary hypertension in the absence of severe valvular disease, and left ventricular systolic dysfunction in which myocardial ischemia has not been excluded. ${ }^{16,33}$ Specific testing modalities for certain cardiac diagnoses such as sarcoidosis, amyloidosis, and hypertrophic cardiomyopathy are included. ${ }^{32}$ Although certain modalities are very specific for diagnoses such as sarcoidosis or amyloidosis, ${ }^{34-37}$ cardiac MR was ranked Appropriate and of significant diagnostic utility across a variety of disease states. The evaluation of the aortic sinuses and ascending aorta are well-delineated by cardiac MR, cardiac CT, and transesophageal echocardiography when transthoracic echo has not proven definitive. ${ }^{20,21}$ All 3 modalities were ranked Appropriate. Likewise, cardiac MR and cardiac CT are useful beyond coronary angiography for further anatomic characterization of anomalous coronary arteries.

Table 4 evaluates sequential or follow-up testing to assess for clinical stability when a diagnosis has been established and the patient is asymptomatic or exhibits stable symptoms. All modalities were found to be Rarely Appropriate when used for repeat imaging in less than 1 year in patients at risk of heart failure without structural heart disease, with known hypertension without a change in their clinical status, or with systolic or diastolic heart failure without a change in clinical status. ${ }^{18}$ Conversely, in patients who are imaged after having undergone therapy with potentially cardiotoxic agents, repeat imaging in less than a year was deemed Appropriate for transthoracic echo, strain imaging, and radionuclide ventriculography, and May Be Appropriate for cardiovascular magnetic resonance imaging. ${ }^{38,39}$ In patients with a bicuspid aortic valve with an initial aortic dilatation of greater than 4.5 centimeters, family history of dissection or rapid rate of change in the aortic diameter, re-evaluation in less than 1 year by cardiovascular magnetic resonance imaging or $\mathrm{CT}$ is rated May Be Appropriate. There was discussion among writing group members that evaluation by transthoracic echo may be considered in a specific subset of patients in which the involved segment of the aorta is well-visualized by echo and/or in which repeated exposure to radiation is undesirable, such as in young women.

In patients without these concerning features, imaging in less than 1 year is considered Rarely Appropriate for all modalities. ${ }^{20}$ Evaluation of known moderate or greater pulmonary hypertension by transthoracic echo in an interval of less than 1 year was rated May Be Appropriate, whereas re-evaluation after 1 year or longer was deemed Appropriate. Serial imaging of a chronic asymptomatic pericardial effusion in instances in which findings would potentially alter therapy was rated Appropriate for transthoracic echo and May Be Appropriate for cardiac MR. In a similar fashion, re-evaluation of an intracardiac mass when findings would potentially alter therapy was deemed Appropriate for transthoracic and transesophageal echocardiography, cardiac MR, and cardiac CT. It is not recommended to re-evaluate for resolution of left atrial thrombus after anticoagulation unless a change in therapy is warranted. In this instance, all modalities were rated Rarely Appropriate.

Table 5 evaluates sequential or follow-up testing in which a diagnosis has been established in the setting of new or worsening symptoms or to guide therapy. This table encompasses a variety of clinical diagnoses. It includes patients with heart failure with a deterioration in their clinical status and re-evaluation after revascularization or optimal medical therapy to determine device candidacy. It also includes reevaluation for cardiac resynchronization therapy device optimization. Serial imaging was also used to evaluate the progression of a pericardial effusion with the development of tamponade or the progression of constrictive pericarditis. Serial imaging was also use to evaluate patients with known aortic dilatation with a change in clinical status. In this table, a variety of imaging modalities showed utility. Although transthoracic echo was generally ranked Appropriate for most of these scenarios, there was a significant role for transesophageal echo in specific indications such as evaluation of the thoracic aorta and resolution of intracardiac thrombus. Cardiac MR and CT were also useful for a variety of indications. Cardiac CT was rated Appropriate for evaluation of pericardial mass and the thoracic aorta as was cardiac MR, whereas cardiac MR was found to be Appropriate for re-evaluation of the progression of constriction. Radionuclide ventriculography was Appropriate for serial evaluation of patients undergoing cardiotoxic therapy, where it was ranked Appropriate. It was ranked May Be Appropriate for serial imaging in patients with known cardiomyopathy with a change in their clinical status.

Section 6.3. evaluates cardiac structure and function in patients undergoing transcatheter interventions for structural heart disease. Table 6 evaluates imaging for the evaluation of transient ischemic attacks or ischemic stroke. ${ }^{40}$ This table is included as a prelude to the subsequent tables on evaluation of closure of a patent foramen ovale or atrial septal defect or structural intervention to place a left atrial appendage occlusion device. Depending upon the clinical suspicion of the source of the stroke or TIA, different modalities are evaluated. Assessment of the intracranial arteries is most appropriately done with MR angiography or CT angiography of the head and neck, whereas assessment of the extracranial arteries is most appropriate with carotid Doppler in addition to these 2 modalities.

The evaluation for closure of a patent foramen ovale or atrial septal defect is divided into preprocedural 
evaluation, ${ }^{41}$ intraprocedural guidance, and assessment following closure of PFO or atrial septal defect (Tables 7-9). For preprocedural guidance, both transthoracic and transesophageal echo are rated Appropriate, and the addition of 3-dimensional (3D) transesophageal echo imaging as an adjunct is rated May Be Appropriate. For assessment of atrial septal anatomy, transthoracic echo, transesophageal echo, 3D transesophageal echo imaging, $\mathrm{CT}$, and MR are rated Appropriate. For intraprocedural guidance, the same imaging tools are deemed appropriate regardless of whether the patient appears to have complex or simple anatomy. In cases requiring intraprocedural guidance, transesophageal echo with or without 3D imaging, intracardiac echo, and fluoroscopy are all rated Appropriate. Routine assessment following closure of a patent foramen ovale is accomplished with transthoracic echo with the occasional use of transesophageal echo, which was rated May Be Appropriate. ${ }^{42}$ Nonroutine assessment of such patients when there is clinical concern for infection, malposition, embolization, or persistent shunt involves utilizing transesophageal echo as a first-line technique where it is ranked Appropriate.

Tables 10-12 evaluate the preprocedural, ${ }^{43,44}$ intraprocedural, ${ }^{45}$ and postprocedural assessment of patients undergoing left atrial appendage occlusion device placement. Evaluation involves the use of both transthoracic and transesophageal echocardiography (with or without 3D), $\mathrm{CT}$ angiography, and, to a lesser extent, cardiac MR angiography. Intraprocedural evaluation involves the use of transesophageal echocardiography (with or without 3D), fluoroscopy, intracardiac echo, and transthoracic echo to screen for early procedural complications. Assessment after deployment of the device involves a transthoracic echo, which may be done prior to discharge. U.S. Food and Drug Administration-mandated surveillance at 45 days includes transthoracic echocardiography, which is rated as May Be Appropriate, and transesophageal echocardiography, which is rated as Appropriate. In long-term follow-up, both transthoracic echo (with or without 3D) and transesophageal echo (with or without 3D) are rated May Be Appropriate.

\section{CONCLUSIONS}

This document assesses a wide array of imaging modalities available to the clinician in the evaluation of patients with non-VHD. Presented here is a broad spectrum of clinical scenarios in such patients. Some of these scenarios replicate those of prior documents, but many are new, specifically structural interventions that were not in the armamentarium of clinicians when prior, single-modality documents were published. The writing group especially wants to thank the rating panel, which helped us clarify the language of many scenarios and which, with focused rerating of a handful of indications, helped us to create a document that is consistent with and supported by medical evidence and helps guide clinicians where evidence is incomplete.

We believe the multimodality approach more closely replicates clinical decision making and will be useful. Future documents will not provide single-source guidance for appropriateness of a single imaging modality in all disease states. Echocardiography indications, for example, will be spread across complimentary documents such as the multimodality stable ischemic heart disease AUC, the valvular heart disease multimodality document, the current document, and the multimodality imaging in pre-operative evaluation document, which is under development.

As with prior documents, the evaluation is a product of current ACC/AHA clinical practice guidelines where available, subspecialty societal guidelines, consensus documents, single-center studies, and expert consensus. ACC/ AHA guidelines are considered the highest level of evidence for the purpose of these efforts but are not available for all scenarios. The modalities are not to be considered in a rank order and may be used relative to individual patient circumstances and the balance of risk versus benefit. Accordingly, a study rated May Be Appropriate should not be denied reimbursement in lieu of one rated Appropriate. In some circumstances, a study ranked Rarely Appropriate may be clinically useful if properly documented.

The American College of Cardiology is recognized as a Qualified Provider-Led Entity in the crafting of these documents and is so recognized by the Center for Medicare and Medicaid Services (CMS). This process demands a rigorous evidentiary review and a commitment to periodic updates.

\section{ACC PRESIDENT AND STAFF}

C. Michael Valentine, MD, FACC, President

Timothy W. Attebery, Chief Executive Officer, MBA, FACHE

William J. Oetgen, MD, FACC, Executive Vice President, Science, Education, Quality, and Publishing

Joseph M. Allen, MA, Team Leader, Clinical Policy and Pathways

Amy Dearborn, Team Leader, Pathways and Appropriate Use Criteria

María Velásquez, Senior Research Specialist, Appropriate Use Criteria

Amelia Scholtz, PhD, Publications Manager, Science, Education, Quality, and Publishing

\section{References}

1. Doherty JU, Kort S, Mehran R, et al. ACC/AATS/AHA/ASE/ASNC/HRS/SCAI/ SCCT/SCMR/STS 2017 appropriate use criteria for multimodality imaging in valvular heart disease: a report of the American College of Cardiology Appropriate Use Criteria Task Force, American Association for Thoracic Surgery, American Heart Association, American Society of Echocardiography, American Society of Nuclear Cardiology, Heart Rhythm Society, Society for Cardiovascular Angiography and Interventions, Society of Cardiovascular Computed Tomography, Society for Cardiovascular Magnetic Resonance, and Society of Thoracic Surgeons. J Am Coll Cardiol. 2017;70:1647-72. 
2. Patel MR, Spertus JA, Brindis RG, et al. ACCF proposed method for evaluating the appropriateness of cardiovascular imaging. J Am Coll Cardiol. 2005;46: 1606-13.

3. Hendel RC, Lindsay BD, Allen JM, et al. ACC appropriate use criteria methodology: 2018 update. J Am Coll Cardiol. 2018;71:935-48.

4. Fitch K, Bernstein SJ, Aguilar MD, et al. The RAND/UCLA Appropriateness Method User's Manual. Arlington, Va: RAND; 2001.

5. Hirshfeld JW Jr, Ferrari VA, Bengel FM, et al. 2018 ACC/HRS/NASCI/SCAI/ SCCT expert consensus document on optimal use of ionizing radiation in cardiovascular imaging: best practices for safety and effectiveness: a report of the American College of Cardiology Task Force on Clinical Expert Consensus Documents. J Am Coll Cardiol. 2018;71:e283-351.

6. Fihn SD, Blankenship JC, Alexander KP, et al. 2014 ACC/AHA/AATS/PCNA/ SCAI/STS focused update of the Guideline for the Diagnosis and Management of Patients With Stable Ischemic Heart Disease: a report of the American College of Cardiology/American Heart Association Task Force on Practice Guidelines, and the American Association for Thoracic Surgery, Preventive Cardiovascular Nurses Association, Society for Cardiovascular Angiography and Interventions, and Society of Thoracic Surgeons. J Am Coll Cardiol. 2014;64:1929-49.

7. Nishimura RA, Otto CM, Bonow RO, et al. 2014 AHA/ACC guideline for the management of patients with valvular heart disease: a report of the American College of Cardiology/American Heart Association task force on practice guidelines. J Am Coll Cardiol. 2014;63:e57-185.

8. Nishimura RA, Otto CM, Bonow RO, et al. 2017 AHA/ACC focused update of the 2014 AHA/ACC Guideline for the Management of Patients With Valvular Heart Disease: a report of the American College of Cardiology/American Heart Association Task Force on Clinical Practice Guidelines. J Am Coll Cardiol. 2017; 70:252-89.

9. Hunt SA, Abraham WT, Chin MH, et al. 2009 focused update incorporated into the ACC/AHA 2005 Guidelines for the Diagnosis and Management of Heart Failure in Adults: a report of the American College of Cardiology Foundation/American Heart Association Task Force on Practice Guidelines developed in collaboration with the International Society for Heart and Lung Transplantation. J Am Coll Cardiol. 2009:53:e1-90.

10. Wolk MJ, Bailey SR, Doherty JU, et al. ACCF/AHA/ASE/ASNC/HFSA/HRS/ SCAI/SCCT/SCMR/STS 2013 multimodality appropriate use criteria for the detection and risk assessment of stable ischemic heart disease: a report of the American College of Cardiology Foundation Appropriate Use Criteria Task Force, American Heart Association, American Society of Echocardiography, American Society of Nuclear Cardiology, Heart Failure Society of America, Heart Rhythm Society, Society for Cardiovascular Angiography and Interventions, Society of Cardiovascular Computed Tomography, Society for Cardiovascular Magnetic Resonance, and Society of Thoracic Surgeons. J Am Coll Cardiol. 2013;63:380-406

11. Hendel RC, Patel MR, Kramer CM, et al. ACCF/ACR/SCCT/SCMR/ASNC/ NASCI/SCAI/SIR 2006 appropriateness criteria for cardiac computed tomography and cardiac magnetic resonance imaging.of cardiovascular imaging: a report of the American College of Cardiology Foundation Quality Strategic Directions Committee Appropriateness Criteria Working Group, American College of Radiology, Society of Cardiovascular Computed Tomography, Society for Cardiovascular Magnetic Resonance, American Society of Nuclear Cardiology, North American Society for Cardiac Imaging, Society for Cardiovascular Angiography and Interventions, and Society of Interventional Radiology. J Am Coll Cardiol. 2006;48:1475-97.

12. Taylor AJ, Cequeira M, Hodgson JM, et al. ACCF/SCCT/ACR/AHA/ASE/ASNC/ NASCI/SCAI/SCMR 2010 appropriate use criteria for cardiac computed tomography: a report of the American College of Cardiology Foundation Appropriate Use Criteria Task Force, the Society of Cardiovascular Computed Tomography, the American College of Radiology, the American Heart Association, the American Society of Echocardiography, the American Society of Nuclear Cardiology, the Society for Cardiovascular Angiography and Interventions, and the Society for Cardiovascular Magnetic Resonance. J Am Coll Cardiol. 2010;56:1864-94.

13. Hendel RC, Berman DS, Di Carli MF, et al. ACCF/ASNC/ACR/AHA/ASE/ SCCT/SCMR/SNM 2009 appropriate use criteria for cardiac radionuclide imaging: a report of the American College of Cardiology Foundation Appropriate Use Criteria Task Force, the American Society of Nuclear Cardiology, the American College of Radiology, the American Heart Association, the American Society of Echocardiography, the Society of Cardiovascular Computed Tomography, the Society for Cardiovascular Magnetic Resonance, and the Society of Nuclear Medicine. J Am Coll Cardiol. 2009;53:2201-29.
14. Douglas PS, Garcia MJ, Haines DE, et al. ACCF/ASE/AHA/ASNC/ HFSA/HRS/ SCAI/SCCM/SCCT/SCMR 2011 appropriate use criteria for echocardiography: a report of the American College of Cardiology Foundation Appropriate Use Criteria Task Force, American Society of Echocardiography, American Heart Association, American Society of Nuclear Cardiology, Heart Failure Society of America, Heart Rhythm Society, Society for Cardiovascular Angiography and Interventions, Society of Critical Care Medicine, Society of Cardiovascular Computed Tomography, and Society for Cardiovascular Magnetic Resonance. J Am Coll Cardiol. 2011;57:1126-66.

15. Greenland P, Alpert JS, Beller GA, et al. 2010 ACCF/AHA guideline for assessment of cardiovascular risk in asymptomatic adults: a report of the American College of Cardiology Foundation/American Heart Association task force on practice guidelines. J Am Coll Cardiol. 2010;56:e50-103.

16. Gersh BJ, Maron BJ, Bonow RO, et al. 2011 ACCF/AHA guideline for the diagnosis and treatment of hypertrophic cardiomyopathy: a report of the American College of Cardiology Foundation/American Heart Association task force on practice guidelines. J Am Coll Cardiol. 2011;58:e212-60.

17. Plana JC, Galderisi M, Barac A, et al. Expert consensus for multimodality imaging evaluation of adult patients during and after cancer therapy: a report from the American Society of Echocardiography and the European Association of Cardiovascular Imaging. J Am Soc Echocardiogr. 2014;27:911-39.

18. Yancy CW, Jessup M, Bozkurt B, et al. 2013 ACCF/AHA guideline for the management of heart failure: a report of the American College of Cardiology Foundation/American Heart Association task force on practice guidelines. J Am Coll Cardiol. 2013;62:e147-239.

19. Maron BJ, Zipes DP, Kovacs RJ, et al, on behalf of the American Heart Association Electrocardiography and Arrhythmias Committee of the Council on Clinical Cardiology, Council on Cardiovascular Disease in the Young, Council on Cardiovascular and Stroke Nursing, Council on Functional Genomics and Translational Biology, and the American College of Cardiology. Eligibility and disqualification recommendations for competitive athletes with cardiovascular abnormalities: preamble, principles, and general considerations: a scientific statement from the American Heart Association and American College of Cardiology. J Am Coll Cardiol. 2015;66:2343-9.

20. Hiratzka LF, Bakris GL, Beckman JA, et al. 2010 ACCF/AHA/AATS/ACR/ ASA/SCA/SCAI/SIR/STS/SVM guidelines for the diagnosis and management of patients with thoracic aortic disease: a report of the American College of Cardiology Foundation/American Heart Association Task Force on Practice Guidelines, American Association for Thoracic Surgery, American College of Radiology, American Stroke Association, Society of Cardiovascular Anesthesiologists, Society for Cardiovascular Angiography and Interventions, Society of Interventional Radiology, Society of Thoracic Surgeons, and Society for Vascular Medicine (developed in collaboration with the American College of Emergency Physicians). J Am Coll Cardiol. 2010;55:e27-129.

21. Warnes CA, Williams RG, Bashore TM, et al. ACC/AHA 2008 guidelines for the management of adults with congenital heart disease: a report of the American College of Cardiology/American Heart Association Task Force on Practice Guidelines (Writing Committee to Develop Guidelines for the Management of Adults With Congenital Heart Disease). J Am Coll Cardiol. 2008;52:e143-263.

22. Cheitlin MD, Armstrong WF, Aurigemma GP, et al. ACC/AHA/ASE 2003 guideline update for the clinical application of echocardiography-summary article: a report of the American College of Cardiology/American Heart Association Task Force on Practice Guidelines (ACC/AHA/ASE Committee to Update the 1997 Guidelines on the Clinical Application of Echocardiography). J Am Coll Cardiol. 2003;42:954-70.

23. Priori SG, Blomstrom-Lundqvist C, Mazzanti A, et al. 2015 ESC guidelines for the management of patients with ventricular arrhythmias and the prevention of sudden cardiac death: The Task Force for the Management of Patients with Ventricular Arrhythmias and the Prevention of Sudden Cardiac Death of the European Society of Cardiology. EP Eurospace. 2015;17:1601-87.

24. January CT, Wann LS, Alpert JS, et al. 2014 AHA/ACC/HRS guideline for the management of patients with atrial fibrillation: a report of the American College of Cardiology/American Heart Association Task Force on Practice Guidelines and the Heart Rhythm Society. J Am Coll Cardiol. 2014;64:e1-76.

25. Bouas-Mosquera A, Peteiro J, Broullon F, et al. Prognostic value of exercise echocardiography in patients with atrial fibrillation. Eur J Echocardiogr. 2010; 11:346-51.

26. Moya A, Sutton R, Ammirati F, et al; Task Force for the Diagnosis and Management of Syncope, European Society of Cardiology. Guidelines for the diagnosis and management of syncope-update 2009. Eur Heart J. 2009;30:2631-71. 
27. Porter TR, Shillcutt SK, Adams MS, et al. Guidelines for the use of echocardiography as a monitor for therapeutic interventions in adults: a report from the American Society of Echocardiography. J Am Soc Echocardiogr. 2015;28:40-56.

28. Spencer K, Kimura BJ, Korcarz CE, et al. Focused cardiac ultrasound: recommendations from the American Society of Echocardiography. J Am Soc Echocardiogr. 2013;26:567-81.

29. Silvestry FE, Kerber RE, Brook MM, et al. Echocardiography-guided interventions. J Am Soc Echocardiogr. 2009;22:213-31.

30. Gorcsan J III, Abraham T, Agler DA. Echocardiography for cardiac resynchronization therapy: recommendations for performance and reporting: a report from the American Society of Echocardiography Dyssynchrony Writing Group. J Am Soc Echocardiogr. 2008;21:191-213.

31. Stainback RF, Estep JD, Agler DA, et al; for the American Society of Echocardiography. Echocardiography in the management of patients with left ventricular assist devices: recommendations of the American Society of Echocardiography. J Am Soc Echocardiogr. 2015;28:853-909.

32. Klocke FJ, Baird MG, Lorell BH, et al. ACC/AHA/ASNC guidelines for the clinical use of cardiac radionuclide imaging - executive summary: a report of the American College of Cardiology/American Heart Association Task Force on Practice Guidelines (ACC/AHA/ASNC Committee to Revise the 1995 Guidelines for the Clinical Use of Radionuclide Imaging). J Am Coll Cardiol. 2003; 42:1318-33.

33. Nagueh SF, Bierig SM, Budoff MJ, et al. Guidelines and standards. American Society of Echocardiography clinical recommendations for multimodality cardiovascular imaging of patients with hypertrophic cardiomyopathy. J Am Soc Echocardiogr. 2011;24:473-98.

34. Youssef G, Leung E, Mylonas I, et al. The use of 18F-FDG PET in the diagnosis of cardiac sarcoidosis: a systematic review and metaanalysis including the Ontario experience. J Nucl Med. 2012;53:241-8.

35. Nadel J, Lancefield T, Voskoboinik A, Taylor AJ. Late gadolinium enhancement identified with cardiac magnetic resonance imaging in sarcoidosis patients is associated with long-term ventricular arrhythmia and sudden cardiac death. Eur Heart J Cardiovasc Imaging. 2015;16:634-41.

36. Ruberg FL, Berk JL. Transthyretin (TTR) cardiac amyloidosis. Circulation. 2012;126:1286-300.
37. Fontana M, Pica S, Reant P, et al. Prognostic value of late gadolinium enhancement cardiovascular magnetic resonance in cardiac amyloidosis. Circulation. 2015;132:1570-9.

38. Schwartz RG, Jain D, Storozynsky E. Traditional and novel methods to assess and prevent chemotherapy-related cardiac dysfunction noninvasively. $\mathrm{J} \mathrm{Nucl}$ Cardiol. 2013:20:443-64.

39. Plana JC, Galderisi M, Barac A, et al. Expert consensus for multimodality imaging: evaluation of adult patients during and after cancer therapy: a report from the American Society of Echocardiography and the European Association of Cardiovascular Imaging. J Am Soc Echocardiogr. 2014;27:911-39.

40. Pristipino C, Anzola GP, Ballerini L, et al. Management of patients with patent foramen ovale and cryptogenic stroke: a collaborative, multidisciplinary, position paper: executive summary. Catheter Cardiovasc Interv. 2013;82: 122-9.

41. Osawa K, Miyoshi T, Morimitsu Y, et al. Comprehensive assessment of morphology and severity of atrial septal defects in adults by CT. J Cardiovasc Comput Tomogr. 2015:9:354-61.

42. Silvestry FE, Cohen MS, Armsby LB, et al. Guidelines for the echocardiographic assessment of atrial septal defect and patent foramen ovale: from the American Society of Echocardiography and Society for Cardiac Angiography and Interventions. J Am Soc Echocardiogr. 2015;28:910-58.

43. Masoudi FA, Calkins H, Kavinsky CJ, et al. 2015 ACC/HRS/SCAI left atrial appendage occlusion device societal overview. J Am Coll Cardiol. 2015;66: 1497-513.

44. Wunderlich NC, Beigel R, Swaans MJ, Ho SY, Siegel RJ. Percutaneous interventions for left atrial appendage exclusion: options, assessment, and imaging using 2D and 3D echocardiography. J Am Coll Cardiol Img. 2015;8: 472-88.

45. Berti S, Paradossi U, Meucci F, et al. Periprocedural intracardiac echocardiography for left atrial appendage closure: a dual-center experience. J Am Coll Cardiol Intv. 2014;7:1036-44.

Key Words: ACC Appropriate Use Criteria, imaging, multimodality, structural heart disease 
APPENDIX A. ACC/AATS/AHA/ASE/ASNC/HRS/SCAI/SCCT/SCMR/STS 2019 APPROPRIATE USE CRITERIA FOR MULTIMODALITY IMAGING IN THE ASSESSMENT OF CARDIAC STRUCTURE AND FUNCTION IN NONVALVULAR HEART DISEASE: MEMBERS OF THE WRITING GROUP, RATING PANEL, INDICATION REVIEWERS, AND AUC TASK FORCE-RELATIONSHIPS WITH INDUSTRY AND OTHER ENTITIES (RELEVANT)

\begin{tabular}{|c|c|c|c|c|c|c|c|c|}
\hline Participant & Employment & Representing & Consultant & $\begin{array}{c}\text { Speakers } \\
\text { bureau }\end{array}$ & $\begin{array}{c}\text { Ownership/ } \\
\text { partnership/ } \\
\text { principal }\end{array}$ & Personal research & $\begin{array}{c}\text { Institutional, } \\
\text { organizational, or other } \\
\text { financial benefit }\end{array}$ & $\begin{array}{l}\text { Expert } \\
\text { witness }\end{array}$ \\
\hline \multicolumn{9}{|c|}{ Writing Group } \\
\hline John U. Doherty & $\begin{array}{l}\text { Thomas Jefferson } \\
\text { University—Professor } \\
\text { of Medicine }\end{array}$ & $\mathrm{ACC}$ & None & None & None & None & None & None \\
\hline Smadar Kort & $\begin{array}{l}\text { Stony Brook University } \\
\text { Medical Center- } \\
\text { Clinical Professor of } \\
\text { Medicine }\end{array}$ & ASE & None & None & None & None & None & None \\
\hline Roxana Mehran & $\begin{array}{l}\text { Mount Sinai Medical } \\
\text { Center-Professor of } \\
\text { Medicine }\end{array}$ & SCAI & $\begin{array}{l}\text { - AstraZeneca } \\
\text { Pharmaceuticals } \\
\text { - Boston Scientific } \\
\text { - Cardiovascular } \\
\text { Systems Inc } \\
\text { - Medscape } \\
\text { - Merck \& Co., Inc. } \\
\text { - Shanghai Bracco } \\
\text { Sine Pharmaceutical } \\
\text { Corp. } \\
\text { - The Medicines } \\
\text { Company }\end{array}$ & None & None & $\begin{array}{l}\text { - Abbott Vascular* } \\
\text { - AstraZeneca } \\
\text { Pharmaceuticals* } \\
\text { - AUM } \\
\text { Cardiovascular* } \\
\text { - Bayer Healthcare } \\
\text { Pharmaceuticals* } \\
\text { - Beth Israel Deaconess } \\
\text { Medical Center* } \\
\text { - Bristol-Myers } \\
\text { Squibb* } \\
\text { - CSL Behring* } \\
\text { - Eli Lilly/DSI* } \\
\text { - Medtronic* } \\
\text { - Novartis } \\
\text { Pharmaceuticals } \dagger \\
\text { - OrbusNeich } \dagger \\
\text { - The Medicines } \\
\text { Company* } \\
\text { - Watermark } \\
\text { Research Partners* }\end{array}$ & $\begin{array}{l}\text { Janssen } \\
\text { Pharmaceuticals, Inc. } \\
\text { (Executive } \\
\text { Committee) } \\
\text { - Osprey Medical } \\
\text { (Executive } \\
\text { Committee) } \\
\text { - WebMD, (Interviews) } \\
\text { - Wiley Blackwell } \\
\text { Publishing Company, } \\
\text { (Book Royalty) } \\
\text { - SCAI, (Officer) } \\
\end{array}$ & None \\
\hline
\end{tabular}




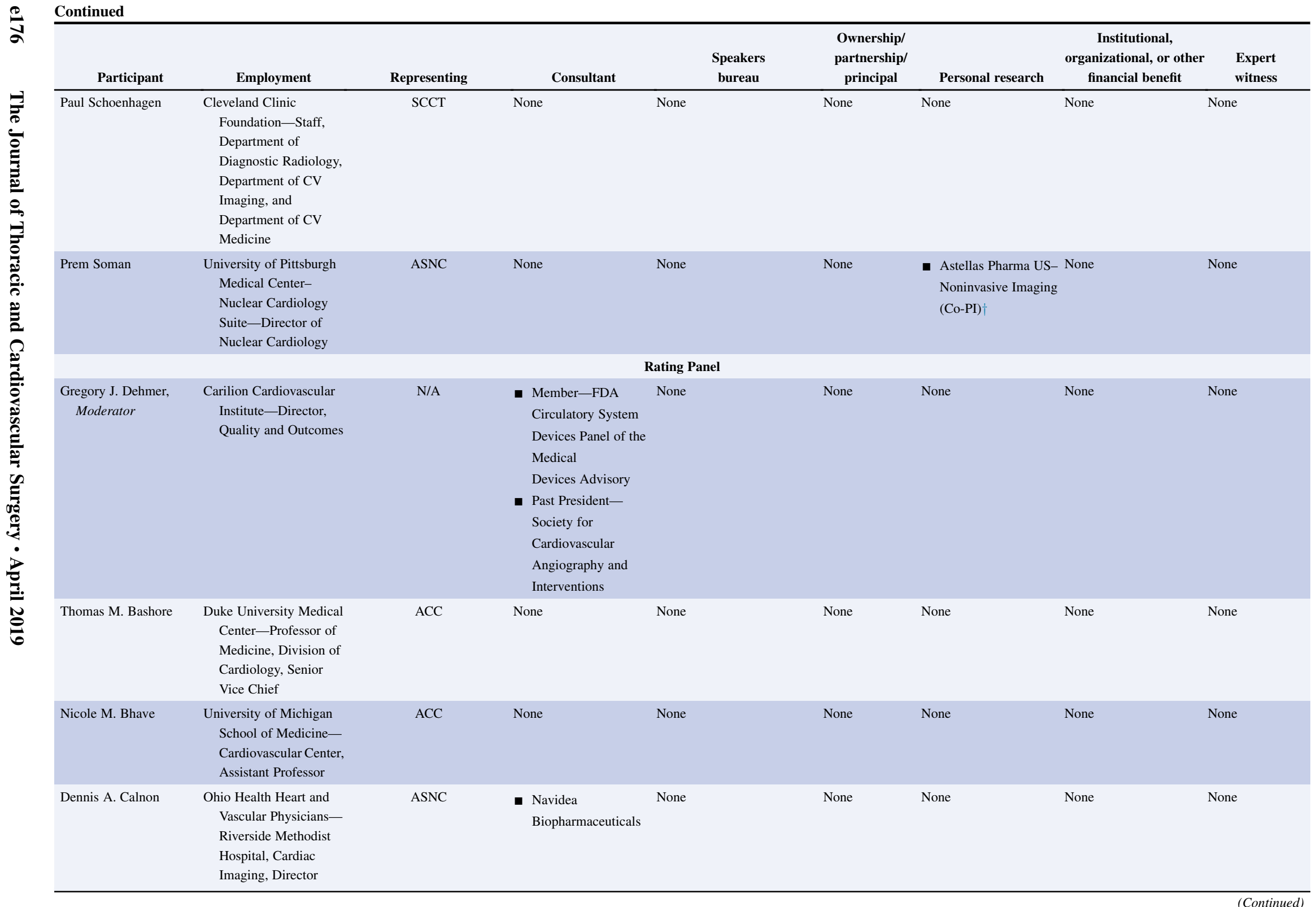




\begin{tabular}{|c|c|c|c|c|c|c|c|c|c|}
\hline Participant & Employment & Representing & Consultant & & $\begin{array}{c}\text { Speakers } \\
\text { bureau }\end{array}$ & $\begin{array}{c}\text { Ownership/ } \\
\text { partnership/ } \\
\text { principal }\end{array}$ & Personal research & $\begin{array}{l}\text { Institutional, } \\
\text { organizational, or other } \\
\text { financial benefit }\end{array}$ & $\begin{array}{l}\text { Expert } \\
\text { witness }\end{array}$ \\
\hline Blase Carabello & $\begin{array}{l}\text { East Carolina } \\
\text { University-Chief, } \\
\text { Division of } \\
\text { Cardiology }\end{array}$ & ACC & None & None & & None & - Edwards (DSMB)* & None & None \\
\hline John V. Conte & $\begin{array}{l}\text { Johns Hopkins School of } \\
\text { Medicine, Division of } \\
\text { Cardiac Surgery- } \\
\text { Director of } \\
\text { Mechanical } \\
\text { Circulatory Support } \\
\text { Professor of Surgery }\end{array}$ & STS & None & None & & None & - Medtronic & $\begin{array}{ll}\text { - } & \text { Medtronic } \\
& \text { (Surgical Advisory } \\
& \text { Board) } \\
\text { - } & \text { Medtronic } \ddagger \\
\text { - } & \text { Boston Scientific } \ddagger\end{array}$ & None \\
\hline Timm Dickfeld & $\begin{array}{l}\text { University of Maryland } \\
\text { School of Medicine- } \\
\text { Professor, Director of } \\
\text { Electrophysiology } \\
\text { Research }\end{array}$ & HRS & $\begin{array}{l}\text { - } \text { Abbott } \dagger \\
\text { - Biosense } \\
\text { - St. Jude } \\
\text { Medical }\end{array}$ & None & & None & $\begin{array}{l}\text { - Biosense } \dagger \\
\text { - General Electric } \dagger\end{array}$ & $\begin{array}{l}\text { Impulse Dynamics } \\
\text { (Event } \\
\text { Adjudication } \\
\text { Committee) } \\
\text { - Siemens-ECG } \\
\text { Reading } \dagger\end{array}$ & $\begin{array}{l}\text { - Plaintiff, } \\
\text { Perforation, } \\
2015 \\
\text { - Plaintiff, } \\
\text { SCD Case, } \\
2015\end{array}$ \\
\hline Dan Edmundowicz & $\begin{array}{l}\text { Temple University } \\
\text { Hospital—Chief, } \\
\text { Section of Cardiology, } \\
\text { Vice Chair of Program } \\
\text { Development, } \\
\text { Professor of Medicine, } \\
\text { Department of } \\
\text { Medicine }\end{array}$ & ACC & None & None & & None & None & None & None \\
\hline Victor A. Ferrari & $\begin{array}{l}\text { Hospital of the University } \\
\text { of Pennsylvania- } \\
\text { Professor of Medicine; } \\
\text { Associate Director, } \\
\text { Cardiovascular } \\
\text { Imaging }\end{array}$ & ACC & None & None & & None & None & $\begin{array}{l}\text { Journal of } \\
\text { Cardiovascular } \\
\text { Magnetic } \\
\text { Resonance } \\
\text { (Officer)* }\end{array}$ & None \\
\hline Michael E. Hall & $\begin{array}{l}\text { University of Mississippi } \\
\text { Medical Center- } \\
\text { Assistant Professor, } \\
\text { Department of } \\
\text { Radiology, Physiology } \\
\text { and Biophysics }\end{array}$ & ACC & None & None & & None & None & None & None \\
\hline Brian Ghoshhajra & $\begin{array}{l}\text { Massachusetts General } \\
\text { Hospital—Service } \\
\text { Chief, Cardiovascular } \\
\text { Imaging, Department }\end{array}$ & SCCT & $\begin{array}{l}\text { - Medtronic } \dagger \\
\text { - Siemens Healthcare } \\
\text { - Research and } \\
\text { Development }\end{array}$ & None & & None & None & None & None \\
\hline
\end{tabular}




\begin{tabular}{|c|c|c|c|c|c|c|c|c|c|}
\hline Participant & Employment & Representing & Consultant & & $\begin{array}{c}\text { Speakers } \\
\text { bureau }\end{array}$ & $\begin{array}{c}\text { Ownership/ } \\
\text { partnership/ } \\
\text { principal }\end{array}$ & Personal research & $\begin{array}{c}\text { Institutional, } \\
\text { organizational, or other } \\
\text { financial benefit }\end{array}$ & $\begin{array}{l}\text { Expert } \\
\text { witness }\end{array}$ \\
\hline & $\begin{array}{l}\text { of Radiology Program; } \\
\text { Director, Cardiac } \\
\text { Imaging Fellowship, } \\
\text { Department of } \\
\text { Radiology }\end{array}$ & & $\begin{array}{l}\text { Institutional } \\
\text { Collaboration }\end{array}$ & & & & & & \\
\hline Praveen Mehrotra & $\begin{array}{l}\text { Thomas Jefferson } \\
\text { University Hospital_- } \\
\text { Assistant Professor of } \\
\text { Medicine, } \\
\text { Echocardiography } \\
\text { Director }\end{array}$ & ACC & None & None & & None & None & None & None \\
\hline Tasneem Z. Naqvi & $\begin{array}{l}\text { Mayo School of } \\
\text { Medicine-Professor } \\
\text { of Medicine, Mayo } \\
\text { Clinic, } \\
\text { Echocardiography } \\
\text { Director; Department } \\
\text { of Cardiovascular } \\
\text { Disease; Consultant }\end{array}$ & ACC & None & None & & None & $\begin{array}{l}\text { - Medtronic* } \\
\text { - St. Jude } \\
\text { Foundation* }\end{array}$ & $\begin{array}{l}\text { Program Director, } \\
\text { Advanced } \\
\text { Echocardiography } \\
\text { Fellowship* } \\
\text { - American Society } \\
\text { of } \\
\text { Echocardiography } \\
\text { (Board Member) }\end{array}$ & None \\
\hline T. Brett Reece & $\begin{array}{l}\text { University of Colorado } \\
\text { Cardiothoracic } \\
\text { Surgery_Associate } \\
\text { Professor, } \\
\text { Cardiothoracic } \\
\text { Surgery; Director, } \\
\text { Comprehensive } \\
\text { Thoracic Aortic } \\
\text { Program }\end{array}$ & AATS & None & None & & None & None & $\begin{array}{l}\text { - Bard } \ddagger \\
\text { - Griols } \ddagger\end{array}$ & None \\
\hline Randall C. Starling & $\begin{array}{l}\text { Cleveland Clinic } \\
\text { Foundation-Head of } \\
\text { Heart Failure and } \\
\text { Cardiac Transplant } \\
\text { Medicine; Medical } \\
\text { Director, Kaufman } \\
\text { Center for Heart } \\
\text { Failure; Staff } \\
\text { Cardiologist, Robert } \\
\text { and Suzanne Tomsich } \\
\text { Department of } \\
\text { Cardiovascular }\end{array}$ & & $\begin{array}{l}\text { - Medtronic } \\
\text { - Novartis }\end{array}$ & None & & $\begin{array}{l}\text { - St. Jude } \\
\text { Medical }\end{array}$ & $\begin{array}{l}\text { - } \text { BioControl (PI) } \\
\text { - } \text { Cardiac } \\
\text { Dimensions (PI)* } \\
\text { - } \text { HeartWare (PI)* } \\
\text { - Medtronic (PI) } \\
\text { - Novartis (PI) } \dagger \\
\text { - } \text { St. Jude } \\
\text { Medical (PI)* }\end{array}$ & None & None \\
\hline
\end{tabular}




\begin{tabular}{|c|c|c|c|c|c|c|c|c|}
\hline Participant & Employment & Representing & Consultant & $\begin{array}{l}\text { Speakers } \\
\text { bureau }\end{array}$ & $\begin{array}{c}\text { Ownership/ } \\
\text { partnership/ } \\
\text { principal }\end{array}$ & Personal research & $\begin{array}{c}\text { Institutional, } \\
\text { organizational, or other } \\
\text { financial benefit }\end{array}$ & $\begin{array}{l}\text { Expert } \\
\text { witness }\end{array}$ \\
\hline & $\begin{array}{l}\text { Medicine; Vice } \\
\text { Chairman, } \\
\text { Cardiovascular } \\
\text { Medicine and } \\
\text { Operations }\end{array}$ & & & & & & & \\
\hline Molly Szerlip & $\begin{array}{l}\text { Baylor Scott and White- } \\
\text { The Heart Hospital } \\
\text { Plano, Medical } \\
\text { Director, Inpatient and } \\
\text { Outpatient Valve } \\
\text { Program; Director, } \\
\text { Structural Heart } \\
\text { Fellowship }\end{array}$ & SCAI & $\begin{array}{l}\text { Edwards } \\
\text { Lifesciences } \\
\text { - Medtronic }\end{array}$ & $\begin{array}{l}\text { - Abiomed } \\
\text { - Edwards Lifesciences }\end{array}$ & None & - Medtronic & $\begin{array}{l}\text { - } \text { Bayer } \ddagger \\
\text { - Edwards } \ddagger \\
\text { - Medtronic } \ddagger\end{array}$ & None \\
\hline Wendy S. Tzou & $\begin{array}{l}\text { University of Colorado } \\
\text { School of Medicine- } \\
\text { Cardiac } \\
\text { Electrophysiology } \\
\text { Section, Division of } \\
\text { Cardiology; Associate } \\
\text { Professor of Medicine }\end{array}$ & AHA & $\begin{array}{l}\text { - } \text { Abbott } \\
\text { Laboratories } \\
\text { - Biosense Webster } \\
\text { - Boston Scientific } \\
\text { - Medtronic }\end{array}$ & None & None & None & None & None \\
\hline John B. Wong & $\begin{array}{l}\text { Tufts University School } \\
\text { of Medicine- } \\
\text { Professor of Medicine }\end{array}$ & $\mathrm{ACC}$ & $\begin{array}{l}\text { Informed Medical } \\
\text { Decisions } \\
\text { Foundation: } \\
\text { Healthwise } \\
\text { - Annals of Internal } \\
\text { Medicine } \\
\text { (American College } \\
\text { of Physicians) }\end{array}$ & None & None & $\begin{array}{l}\text { Patient-Centered } \\
\text { Outreach Institute- } \\
\text { Cardiothoracic } \\
\text { Surgery Congenital } \\
\text { Heart Disease and } \\
\text { Pediatric Cardiology } \\
\text { Invasive CV Angio } \\
\text { and Interventions } \\
\text { Prevention Stable } \\
\text { Ischemic Heart } \\
\text { Disease (PI) } \dagger\end{array}$ & $\begin{array}{l}\text { AMA Physician } \\
\text { Consortium for } \\
\text { Performance } \\
\text { Improvement } \\
\text { (Member, Co-Chair) }\end{array}$ & None \\
\hline & & & & Reviewers & & & & \\
\hline Gurusher S. Panjrath & $\begin{array}{l}\text { George Washington } \\
\text { University—Director, } \\
\text { Heart Failure and } \\
\text { Mechanical Support } \\
\text { Program }\end{array}$ & $\begin{array}{l}\text { ACC-Heart Failure and } \\
\text { Transplant Section } \\
\text { Leadership Council }\end{array}$ & None & - Amgen, Inc. $\dagger$ & None & None & $\begin{array}{l}\text { - Alnylam } \ddagger \\
\text { - CVRx } \ddagger\end{array}$ & None \\
\hline Uma Valeti & $\begin{array}{l}\text { University of } \\
\quad \text { Minnesota-Staff }\end{array}$ & $\begin{array}{l}\text { ACC-Heart Failure and } \\
\text { Transplant Section } \\
\text { Leadership Council }\end{array}$ & None & None & None & $\begin{array}{l}\text { - Bayer-Noninvasive } \\
\text { Imaging (Co-PI) }\end{array}$ & None & None \\
\hline
\end{tabular}




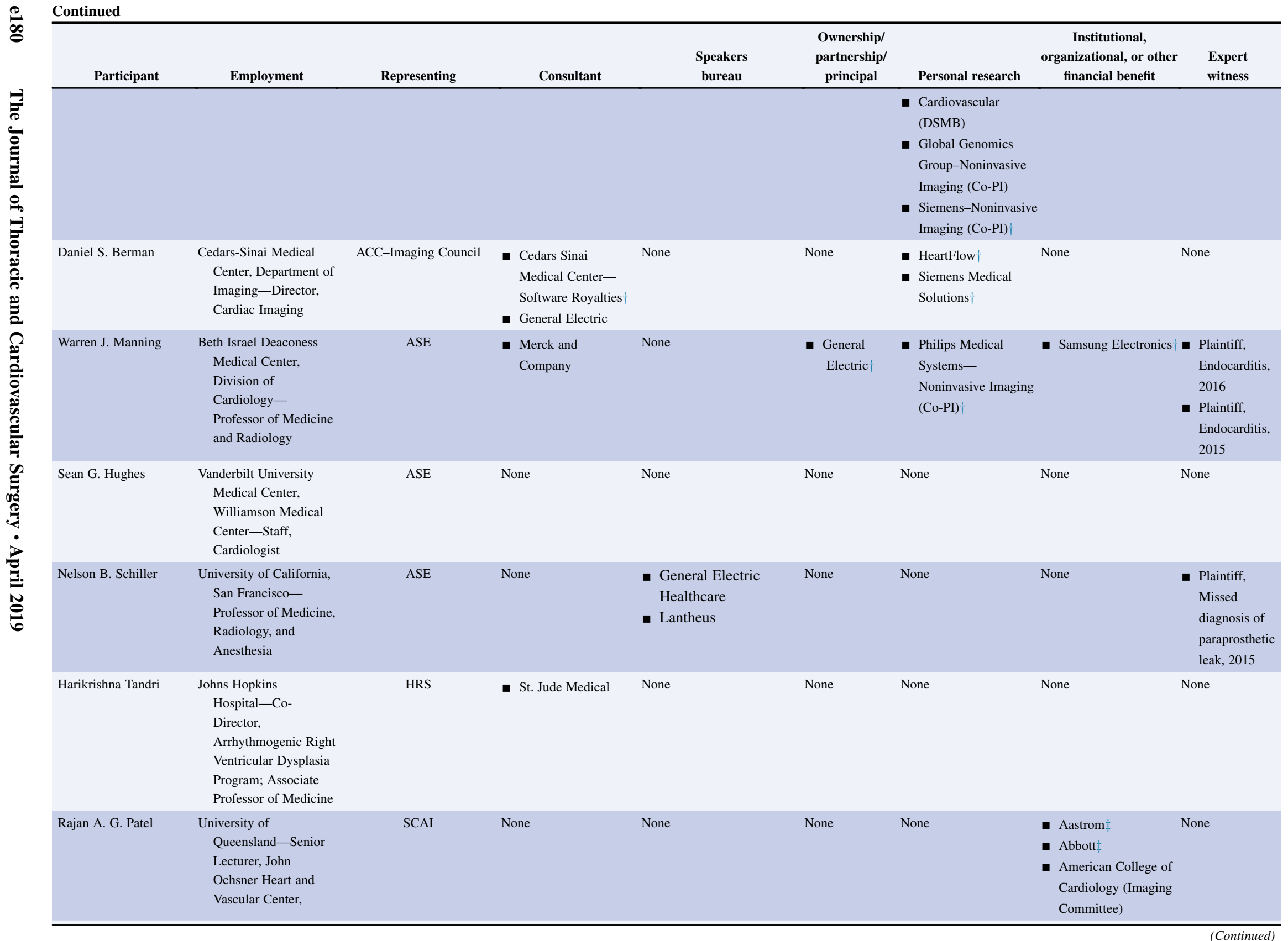




\begin{tabular}{|c|c|c|c|c|c|c|c|c|c|}
\hline Participant & Employment & Representing & Consultant & & $\begin{array}{c}\text { Speakers } \\
\text { bureau }\end{array}$ & $\begin{array}{c}\text { Ownership/ } \\
\text { partnership/ } \\
\text { principal }\end{array}$ & Personal research & $\begin{array}{l}\text { Institutional, } \\
\text { organizational, or other } \\
\text { financial benefit }\end{array}$ & $\begin{array}{l}\text { Expert } \\
\text { witness }\end{array}$ \\
\hline & $\begin{array}{l}\text { Ochsner Medical } \\
\text { Center- } \\
\text { Interventional } \\
\text { Cardiology Section }\end{array}$ & & & & & & & $\begin{aligned} \text { - } & \text { NHLBI } † \\
\text { - } & \text { SCAI Carotid Stent } \\
& \text { (Committee) } \\
\text { - } & \text { SCAI Publications } \\
& \text { (Committee })\end{aligned}$ & \\
\hline Jeffrey A. Brinker & $\begin{array}{l}\text { Johns Hopkins } \\
\text { Hospital—Professor } \\
\text { of Medicine }\end{array}$ & SCAI & None & None & & None & None & None & None \\
\hline Raymond Y. Kwong & $\begin{array}{l}\text { Brigham \& Women's } \\
\text { Hospital Medicine, } \\
\text { Cardiovascular } \\
\text { Division—Instructor } \\
\text { of Medicine }\end{array}$ & SCMR & None & None & & None & $\begin{array}{l}\text { - Alnylam } \\
\text { Pharmaceutical }(\mathrm{PI})_{\dagger}^{\dagger}\end{array}$ & $\begin{array}{l}\text { Society for } \\
\text { Cardiovascular } \\
\text { Magnetic } \\
\text { Resonance } \\
\text { (Officer) }\end{array}$ & None \\
\hline Andrew J. Powell & $\begin{array}{l}\text { Children's Hospital, } \\
\text { Boston, Department of } \\
\text { Cardiology- } \\
\text { Associate in } \\
\text { Cardiology, Associate } \\
\text { Professor of Pediatrics }\end{array}$ & SCMR & None & None & & None & None & None & None \\
\hline Joseph Wu & $\begin{array}{l}\text { Stanford University } \\
\text { School of Medicine- } \\
\text { Director, Stanford } \\
\text { Cardiovascular } \\
\text { Institute; Professor, } \\
\text { Department of } \\
\text { Medicine/ Cardiology }\end{array}$ & AHA & None & None & & $\begin{array}{l}\text { - Stem Cell } \\
\text { Theranostics* }\end{array}$ & None & None & None \\
\hline Harold Litt & $\begin{array}{l}\text { University of } \\
\text { Pennsylvania- } \\
\text { Associate Professor of } \\
\text { Radiology; Chief, } \\
\text { Cardiovascular } \\
\text { Imaging Section, } \\
\text { Department of } \\
\text { Radiology; Director, } \\
\text { Center for Advanced } \\
\text { Computed } \\
\text { Tomography Imaging } \\
\text { Sciences; Fellowship } \\
\text { Director, } \\
\text { Cardiovascular } \\
\text { Imaging Fellowship }\end{array}$ & AHA & None & None & & None & $\begin{array}{ll}\text { - } & \text { American College of } \\
\text { Radiology Imaging } \\
\text { Network- } \\
\text { Noninvasive Imaging } \\
\text { (PI) } \dagger \\
\text { - } \\
\text { HeartFlow- } \\
\text { Noninvasive Imaging } \\
\text { (Co-PI) } \dagger \\
\text { - } \\
\text { Siemens Medical } \\
\text { Solutions- } \\
\text { Noninvasive Imaging } \\
\text { (Co-PI) } \dagger\end{array}$ & None & $\begin{array}{l}\text { Defendant, } \\
\text { Chest mass } \\
\text { imaging } 2016\end{array}$ \\
\hline
\end{tabular}




\begin{tabular}{|c|c|c|c|c|c|c|c|c|c|}
\hline Participant & Employment & Representing & Consultant & & $\begin{array}{c}\text { Speakers } \\
\text { bureau }\end{array}$ & $\begin{array}{c}\text { Ownership/ } \\
\text { partnership/ } \\
\text { principal }\end{array}$ & Personal research & $\begin{array}{l}\text { Institutional, } \\
\text { organizational, or other } \\
\text { financial benefit }\end{array}$ & $\begin{array}{l}\text { Expert } \\
\text { witness }\end{array}$ \\
\hline Thomas C. Gerber & $\begin{array}{l}\text { Mayo Clinic_-Professor } \\
\text { of Medicine and } \\
\text { Radiology }\end{array}$ & AHA & None & None & & None & None & $\begin{array}{l}\text { - American Journal of } \\
\text { Radiology (Officer) } \\
\text { - Mayo Clinic } \\
\text { Proceedings (Officer) }\end{array}$ & None \\
\hline Amish Raval & $\begin{array}{l}\text { University of Wisconsin } \\
\text { School of Medicine- } \\
\text { Associate Professor }\end{array}$ & AHA & None & None & & None & None & None & None \\
\hline Marcelo F. DiCarli & $\begin{array}{l}\text { Brigham and Women's } \\
\text { Hospital-Chief of } \\
\text { Nuclear Medicine; } \\
\text { Harvard Medical } \\
\text { School—Assistant } \\
\text { Professor of } \\
\text { Radiology and } \\
\text { Medicine }\end{array}$ & SNMMI & None & None & & None & $\begin{array}{ll}\text { - } & \text { NHLBI } \\
& \text { T32HL094301- } \\
\text { Noninvasive Imaging } \\
\text { (PI) } \dagger \\
\text { - Spectrum } \\
\text { Dynamics- } \\
\text { Noninvasive Imaging } \\
\text { (Co-PI) } \dagger\end{array}$ & $\begin{array}{l}\text { AHA Circulation: } \\
\text { Cardiovascular } \\
\text { Imaging (Editor) } \\
\text { - } \mathrm{NIH}^{\dagger} \\
\text { - Spectrum Dynamics }{ }^{\dagger}\end{array}$ & None \\
\hline
\end{tabular}

This table represents relevant relationships of participants with industry and other entities that were reported by reviewers at the time this document was under development. The table does not necessarily reflect relationships with industry at the time of publication. A person is deemed to have a significant interest in a business if the interest represents ownership of $\geq 5 \%$ of the voting stock or share of the business entity, or ownership of $\geq \$ 5,000$ of the fair market value of the business entity; or if funds received by the person from the business entity exceed $5 \%$ of the person's gross income for the previous year. Relationships that exist with no financial benefit are also included for the purpose of transparency. Relationships in this table are modest unless otherwise noted. In addition, to ensure complete transparency, a full list of disclosure information-including relationships not pertinent to this document-is available as an Online Appendix. Please refer to http://www.acc.org/guidelines/about-guidelines-and-clinical-documents/relationships-with-industry-policy for definitions of disclosure categories or additional information about the ACC Disclosure Policy for Writing Committees. ACC, American College of Cardiology; AATS, American Association for Thoracic Surgery; $A H A$, American Heart Association; ASE, American Society of Echocardiography; $A S N C$, American Society for Nuclear Cardiology; HRS, Heart Rhythm Society; SCAI, Society for Cardiovascular Angiography and Interventions; SCCT, Society of Cardiovascular Computed Tomography; SCMR, Society for Cardiovascular Magnetic Resonance; STS, Society of Thoracic Surgeons; $A U C$, Appropriate Use Criteria; $A M A$, American Medical Association; $C V$, cardiovascular; $D S M B$, Data and Safety Monitoring Board; $N H L B I$, National Heart, Lung, and Blood Institute; $P I$, Principal Investigator; $S C A$, Society of Cardiovascular Anesthesiologists; $S N M M I$, Society for Nuclear Medicine and Molecular Imaging. *No financial benefit. †SSignificant relationship. ‡Clinical Trial Enroller. 